\title{
DIFFERENTIAL POSETS
}

\author{
RICHARD P. STANLEY
}

\section{INTRODUCTION}

In this paper we introduce a class of partially ordered sets, called differential posets, with many remarkable combinatorial and algebraic properties. (Terminology from lattice theory and the theory of partially ordered sets not explained here may be found in [St6].) The combinatorial properties of differential posets are concerned with the counting of saturated chains $x_{1}<x_{2}<\cdots<x_{k}$ or of "Hasse walks" $x_{1}, x_{2}, \ldots, x_{k}$ (i.e., for $1 \leq i \leq k-1$, either $x_{i+1}$ covers $x_{i}$ or $x_{i}$ covers $x_{i+1}$ ) with various properties. The counting of chains in partially ordered sets is a well-developed subject with many applications both within and outside of combinatorics. For an introduction to this area, see [St6], especially $\S \S 3.5,3.11,3.12,3.13,3.15,3.16,4.5$, and many of the exercises in Chapters 3 and 4. The counting of Hasse walks in a poset is a special case of the counting of walks in a graph. This is the basis for the "transfer-matrix method" [St6, $\S 4.7]$ of enumerative combinatorics, with many applications to probability theory (Markov chains in particular), statistical mechanics, and other areas. See [C-D-S] for additional information.

A basic tool in the theory of differential posets $P$ is the use of two adjoint linear transformations $U$ and $D$ on the vector space of linear combinations of elements of $P$. If $x \in P$ then $U x$ (respectively, $D x$ ) is the sum of all elements covering $x$ (respectively, which $x$ covers). Such linear transformations have appeared in many contexts, but rarely does one have such explicit information as for differential posets. Linear transformations identical or similar to $U$ and/or $D$ appear, for instance, in [P, St5, St7] in order to obtain structural information about $P$; for differential posets the corresponding result is our Corollary 4.4. One may also regard $U$ and $D$ as instances of the finite Radon transform (e.g., $[\mathrm{Ku}]$ ).

For differential posets a fundamental property of $U$ and $D$ is the commutation rule $D U-U D=r I$ for some positive integer $r$ (see Theorem 2.2). Thus differential posets may be regarded as affording a representation of the "Weyl

Received by the editors December 8, 1987.

1980 Mathematics Subject Classification (1985 Revision). Primary 05A15; Secondary 05A17, 06A10, 20C30.

Partially supported by NSF grant \#DMS-8401376. 
algebra" generated by $U$ and $D / r$. This provides an interesting combinatorial realization of Weyl algebras (though we obtain no new theoretical results involving Weyl algebras).

In Theorem 4.1 we compute the spectrum of the operator $U D$ and in Proposition 4.6 its eigenvectors; this result is extended in Proposition 4.12 to more general functions of $U$ and $D$. Besides their intrinsic interest, such results are used in the theory of towers of multimatrix algebras [G-H-J].

The spectrum of $U D$ is closely related to the spectrum (= eigenvalues of the adjacency matrix) of certain finite graphs associated with differential posets. We give (Theorem 4.14) a complete description of such spectra, as well as the corresponding eigenvectors. Thus we have a new class of graphs whose spectra can be explicitly computed. Many interesting properties of a graph are related to its spectrum (such as properties of random walks on the graph); see [C-D-S] for further information.

The prototypical example of a differential poset is Young's lattice $Y$, first studied per se by $\mathrm{G}$. Kreweras $[\mathrm{K}] . Y$ is defined to be the set of all partitions of all nonnegative integers $n$, ordered by inclusion of Young diagrams. Thus if $\lambda=\left(\lambda_{1}, \lambda_{2}, \ldots\right)$ and $\mu=\left(\mu_{1}, \mu_{2}, \ldots\right)$ are partitions (always ordered so $\lambda_{1} \geq \lambda_{2} \geq \cdots$ and $\left.\mu_{1} \geq \mu_{2} \geq \cdots\right)$, then $\mu \leq \lambda$ in $Y$ if and only if $\mu_{i} \leq \lambda_{i}$ for all $i$. We write $\mathbb{N}=\{0,1,2, \ldots\}$ and $\mathbb{P}=\{1,2,3, \ldots\}$. Young's lattice is a locally finite distributive lattice with $\widehat{O}$ (the empty partition $\varnothing$ ); in fact, it is the lattice $J_{f}\left(\mathbb{N}^{2}\right)$ of finite order ideals of the poset $\mathbb{N}^{2}$ (with the usual total ordering of $\mathbb{N}$ ). If $\lambda \in Y$ is a partition of $n$ (i.e., $\sum \lambda_{i}=n$ ), then we write $|\lambda|=n$ or $\lambda \vdash n$. Young's lattice is graded with rank function $\rho$ given by $\rho(\lambda)=|\lambda|$.

Many remarkable enumerative properties of $Y$ are consequences of the theory of symmetric functions, the representation theory of the symmetric group and the complex general linear group, and the Robinson-Schensted correspondence. In particular, a standard Young tableau (SYT) of shape $\lambda$ may be identified with a saturated chain $\phi=\lambda^{0} \subset \lambda^{1} \subset \cdots \subset \lambda^{n}=\lambda$ of partitions from $\phi$ to $\lambda$ (viz., insert the number $i$ in the square $\lambda^{i}-\lambda^{i-1}$, where we are identifying a partition with its diagram [M, pp. 1-2]). Thus, for instance, if $f^{\lambda}$ denotes the number of SYT of shape $\lambda$, then the classical result

$$
\sum_{\lambda \vdash n}\left(f^{\lambda}\right)^{2}=n !
$$

asserts, in terms of Young's lattice, that the number of sequences (or Hasse walks)

$$
\phi=\lambda^{0}<\lambda^{1}<\cdots<\lambda^{n}>\mu^{n-1}>\cdots>\mu^{0}=\phi,
$$

where $\lambda^{i}$ and $\mu^{i}$ are partitions of $i$, is equal to $n$ !. The theory of differential posets shows that such a result depends on only simple structural properties of $Y$ and can be extended to many other posets.

Many of our results on differential posets are new even for Young's lattice. In [S-S] a number of our present results are given combinatorial proofs for the 
case of Young's lattice. We should also mention that several generalizations of differential posets are discussed in [St8], which is a sequel to the present paper.

In general, if $P$ is any graded poset then we let $\rho$ denote its rank function, i.e., if $x \in P$ then $\rho(x)$ is the length $l$ of the longest chain $x_{0}<x_{1}<\cdots<$ $x_{l}=x$ in $P$ with top element $x$. Write

$$
P_{i}=\{x \in P: \rho(x)=i\},
$$

so $P=P_{0} \cup P_{1} \dot{\cup} \cdots$ (disjoint union). We will also use the notation $\# S$ or $|S|$ for the cardinality of the finite set $S$.

1.1. Definition. Let $r$ be a positive integer. A poset $P$ is called $r$-differential if it satisfies the following three conditions:

(D1) $P$ is locally finite and graded and has a $\widehat{O}$ element.

(D2) If $x \neq y$ in $P$ and there are exactly $k$ elements of $P$ which are covered by both $x$ and $y$, then there are exactly $k$ elements of $P$ which cover both $x$ and $y$.

(D3) If $x \in P$ and $x$ covers exactly $k$ elements of $P$, then $x$ is covered by exactly $k+r$ elements of $P$.

If $P$ is an $r$-differential poset for some $r$, then we call $P$ a differential poset.

1.2. Proposition. If $P$ is a poset satisfying (D1) and (D2), then for $x \neq y$ in $P$ the integer $k$ of $(\mathrm{D} 2)$ is equal to 0 or 1 .

Proof. Suppose the contrary. Let $x$ and $y$ be elements of minimal rank for which $k>1$. Thus $x$ and $y$ both cover elements $x_{1} \neq y_{1}$ of $P$. But then $x_{1}$ and $y_{1}$ are elements of smaller rank with $k>1$, a contradiction.

1.3. Proposition. Let $L$ be a lattice satisfying (D1) and (D3). Then $L$ is $r$-differential if and only if $L$ is modular.

Proof. It is well known (e.g., [Bi, Theorem 16, p. 41]) that a locally finite lattice is modular if and only if the following condition is satisfied: For all $x, y \in L$, $x$ and $y$ cover $x \wedge y$ if and only if $x \vee y$ covers $x$ and $y$. But this condition is equivalent to (D2).

1.4. Corollary. Young's lattice $Y$ is 1-differential. More generally, $Y^{r}$ is r-differential.

Proof. It was observed in [St3, p. 225] or [St6, Exercise 3.22(c)] that $Y^{r}$ satisfies (D3). Since $Y^{r}$ is distributive, it is also modular, so by Proposition 1.3 $Y^{r}$ is $r$-differential.

Further examples of differential posets are given in $\S 5$ and in Proposition 6.1 .

\section{TWO LINEAR TRANSFORMATIONS}

Let $K_{0}$ be a field of characteristic 0 . Let $N$ be a "big enough" set of indeterminates (almost always $N=\{q, t\}$ will suffice, but for instance in Proposition 
3.10 we need $\left.N=\left\{q, s_{1}, s_{2}, \ldots, t_{1}, t_{2}, \ldots\right\}\right)$, and let $K$ denote the quotient field of the ring $K_{0}((N))$ of formal Laurent series

$$
F(N)=\sum_{\nu} a_{\nu} \prod_{t \in N} t^{\nu(t)},
$$

where the sum ranges over all functions $\nu: N \rightarrow \mathbb{Z}$, and where $a_{\nu}=0$ if $\nu(t)<n_{t}$ for fixed integers $n_{t}$ (depending on $F$ ). (It would suffice to let $K=K_{0}((N))$, but for convenience we work over a field.)

Given a set $S$, let $K S$ denote the $K$-vector space with basis $S$, and let $\widehat{K} S$ denote the $K$-vector space of arbitrary (i.e., infinite) linear combinations

$$
\sum_{x \in S} c_{x} x, \quad c_{x} \in K,
$$

of elements of $S$. Thus $\widehat{K} S$ is the completion of $K S$ in a suitable topology.

Given a locally finite poset $P$ and $x \in P$, define

$$
\begin{aligned}
& C^{-}(x)=\{y \in P: x \text { covers } y\}, \\
& C^{+}(x)=\{y \in P: \quad y \text { covers } x\} .
\end{aligned}
$$

2.1. Definition. Let $P$ be a locally finite poset such that for all $x \in P$ the sets $C^{-}(x)$ and $C^{+}(x)$ are finite. Define two continuous linear transformations $U, D: \widehat{K} P \rightarrow \widehat{K} P$ by the condition that for $x \in P$,

$$
U x=\sum_{y \in C^{+}(x)} y,
$$

$$
D x=\sum_{y \in C^{-}(x)} y .
$$

Note. Continuity means that $U$ and $D$ preserve infinite linear combinations, while the condition that $C^{-}(x)$ and $C^{+}(x)$ are finite insures that for any $v \in$ $\widehat{K} P$ the coefficient of $x \in P$ in $U v$ and $D v$ is a finite sum. Hence (1) and (2) indeed define $U v$ and $D v$ for any $v \in \widehat{K} P$.

Let $I: \widehat{K} P \rightarrow \widehat{K} P$ denote the identity transformation, i.e., $I v=v$ for all $v \in \widehat{K} P$. We come to two simple but fundamental results.

2.2. Theorem. Let $P$ be a locally finite graded poset with $\widehat{O}$, with finitely many elements of each rank. Let $r$ be a positive integer. The following two conditions are equivalent:

(a) $P$ is $r$-differential.

(b) $D U-U D=r I$.

(Linear transformations operate right-to-left, e.g., $D U v=D(U v)$.) 
Proof. Let $x \in P$. Then $D U x=\sum_{y} c_{y} y$, where $c_{y}=\#\left(C^{+}(x) \cap C^{+}(y)\right)$. Moreover, $U D x=\sum_{y} d_{y} y$, where $d_{y}=\#\left(C^{-}(x) \cap C^{-}(y)\right)$. Hence $D U-U D=$ $r I$ if and only if for all $x, y \in P$,

$$
\#\left(C^{+}(x) \cap C^{+}(y)\right)=\#\left(C^{-}(x) \cap C^{-}(y)\right), \quad \text { if } x \neq y,
$$

and

$$
\# C^{+}(x)=r+\# C^{-}(x) .
$$

But these are precisely the conditions for $P$ to be $r$-differential.

If $S \subseteq P$, then write

$$
\mathbf{S}=\sum_{x \in S} S \in \widehat{K} \boldsymbol{P} .
$$

2.3. Theorem. If $P$ is an $r$-differential poset, then

$$
D \mathbf{P}=(U+r) \mathbf{P} .
$$

Proof. If $D \mathbf{P}=\sum a_{x} x$, then $a_{x}=\# C^{+}(x)$. Similarly, if $(U+r) \mathbf{P}=\sum b_{x} x$, then $b_{x}=r+\# C^{-}(x)$. The result follows from (D3). ((D2) is not needed.)

Given a vector space $V$, let $\operatorname{End}(V)$ denote the ring of all linear transformations $T: V \rightarrow V$. In the results below we will be dealing with certain formal power series in the linear transformations $U, D \in \operatorname{End}(\widehat{K} P)$, where $P$ is always assumed to be an $r$-differential poset. We may regard such formal power series as elements of the ring $K\langle\langle U, D\rangle\rangle /(D U-U D-r)$, where $K\langle\langle U, D\rangle\rangle$ denotes the ring of noncommutative formal power series in $U$ and $D$ over the field $K$. Arbitrary power series need not define elements of $\operatorname{End}(\widehat{K} P)$, e.g., $\sum_{n \geq 0} D^{n}$ or $e^{D}:=\sum_{n \geq 0} D^{n} / n$ !. But most of the power series we deal with will define linear transformations. In particular, we will consider such power series as

$$
\begin{gathered}
G(U)=\sum_{n \geq 0} a_{n} U^{n}, \quad a_{n} \in K, \\
H(U, D t)=\sum_{n \geq 0} A_{n}(U) D^{n} t^{n}, \quad A_{n}(z) \in K_{0}[[z]] .
\end{gathered}
$$

Thus (3) defines an element of $\operatorname{End}(\widehat{K} P)$ because for any $x \in P$ and $v \in \widehat{K} P$ the coefficient of $x$ in $U^{n} v$ is equal to 0 for $n$ sufficiently large. Similarly $H(U, D t) \in \operatorname{End}(\widehat{K} P)$ since for any $x \in P$ and $v \in \widehat{K} P$ the coefficient of $x$ in $H(U, D t) v$ is easily seen to be a well-defined formal power series in the variable $t$ with coefficients in $K_{0}$. (We could make the above discussion more precise by introducing suitable topological notions, but this seems unnecessarily pedantic.)

Given formal power series such as $f(U)=\sum_{n \geq 0} a_{n} U^{n}$ and $H(U, t)=$ $\sum_{n \geq 0} f_{n}(U) t^{n} / n !$, we will use notation from calculus in a formal way, such as

$$
f^{\prime}(U)=\sum_{n \geq 0} n a_{n} U^{n-1}=\sum_{n \geq 0}(n+1) a_{n+1} U^{n}
$$




$$
\begin{gathered}
\frac{\partial H}{\partial U}=H_{U}(U, t)=\sum_{n \geq 0} f_{n}^{\prime}(U) t^{n} / n !, \\
\frac{\partial H}{\partial t}=H_{t}(U, t)=\sum_{n \geq 0} f_{n}(U) n t^{n-1} / n !=\sum_{n \geq 0} f_{n+1}(U) t^{n} / n !, \\
\int_{0}^{U} f(s) d s=\sum_{n \geq 0} a_{n} U^{n+1} /(n+1)=\sum_{n \geq 1} a_{n-1} U^{n} / n,
\end{gathered}
$$

etc.

It will be convenient to introduce a pairing

$$
\langle,\rangle: \widehat{K} P \times K P \rightarrow K
$$

defined by the conditions that the pairing is bilinear, continuous in the first coordinate, and

$$
\langle x, y\rangle=\delta_{x y} \text { for } x, y \in P
$$

Thus in general

$$
\left\langle\sum a_{x} x, \sum b_{x} x\right\rangle=\sum a_{x} b_{x},
$$

which is a finite sum since only finitely many $b_{x} \neq 0$. (We cannot extend $\langle$, to a scalar product on all of $\widehat{K} P$ since in general the infinite sum $\sum a_{x} b_{x}$ is not defined.) Note that $D$ and $U$ are adjoint under this pairing, i.e.,

$$
\langle D f, g\rangle=\langle f, U g\rangle, \quad\langle U f, g\rangle=\langle f, D g\rangle,
$$

since for $x, y \in P$, we have

$$
\langle D x, y\rangle=\langle x, U y\rangle= \begin{cases}1, & \text { if } x \text { covers } y, \\ 0, & \text { otherwise }\end{cases}
$$

and similarly for $\langle U x, y\rangle$ and $\langle x, D y\rangle$.

2.4. Corollary. Suppose $P$ is $r$-differential. Then for any $f(U) \in K[[U]]$ we have

$$
D f(U)=r f^{\prime}(U)+f(U) D .
$$

Moreover, if $f(U)$ defines an element of $\operatorname{End}(\widehat{K} P)$, then

(b) $D f(U) \mathbf{P}=\left(r f^{\prime}(U)+(U+r) f(U)\right) \mathbf{P}$.

Proof. (a) By linearity and continuity it suffices to assume $f(U)=U^{n}, n \geq 0$. It is then straightforward to prove (4) by induction on $n$; the details are left to the reader.

(b) By (a) and Theorem 2.3,

$$
\begin{aligned}
D f(U) \mathbf{P} & =\left(r f^{\prime}(U)+f(U) D\right) \mathbf{P} \\
& =\left(r f^{\prime}(U)+f(U)(r+U)\right) \mathbf{P} .
\end{aligned}
$$


The effect of Corollary 2.4 is that, informally, we can view the operator $D$ as the derivative $\partial / \partial U$. (We use the partial derivative symbol since later we will also be differentiating with respect to indeterminates in $K$.) In this way many enumerative problems will reduce to solving a partial differential equation. This explains our terminology "differential poset."

We now come to our primary tools for deriving enumerative properties of differential posets.

2.5. Theorem. Let $P$ be an $r$-differential poset. Let $f(U), h(U) \in K_{0}[[U]]$ and $c \in K_{0}$. Then

$$
e^{(f(U)+c D) t} h(U)=e^{\int_{0}^{t} f(U+c r s) d s} h(U+c r t) e^{c D t} .
$$

Moreover, if $f(U)$ and $h(U)$ define elements of $\operatorname{End}\left(\widehat{K}_{0} P\right)$, then:

$$
e^{(f(U)+c D) t} h(U) \mathbf{P}=\exp \left[c r t+\frac{1}{2} c^{2} r t^{2}+c U t+\int_{0}^{t} f(U+c r s) d s\right] h(U+c r t) \mathbf{P} .
$$

Proof. (a) Let

$$
H(t)=e^{(f(U)+c D) t} h(U)=\sum_{n \geq 0}(f(U)+c D)^{n} h(U) \frac{t^{n}}{n !} .
$$

Then the operator $H(t)$ is uniquely determined by the conditions

$$
(f(U)+c D) H=H_{t}, \quad H(0)=h(U) .
$$

Hence we need to verify only that the right-hand side of (5) satisfies (8). But this is a formal computation, taking care that $U$ and $D$ do not commute. Specifically, writing $L(t)$ for the right-hand side of (5), we have (using Corollary $2.4(\mathrm{a}))$,

$$
\begin{aligned}
(f(U)+c D) L(t)= & f(U)+c r \int_{0}^{t} f_{U}(U+c r s) d s \\
& \left.+c r h^{\prime}(U+c r t) / h(U+c r t)\right] L(t)+c L(t) D \\
= & f(U)+(f(U+c r t)-f(U)) \\
& \left.+c r h^{\prime}(U+c r t) / h(U+c r t)\right] L(t)+c L(t) D \\
& \left(\text { since } c r f_{U}(U+c r s)=f_{s}(U+c r s)\right) \\
= & {\left[f(U+c r t)+c r h^{\prime}(U+c r t) / h(U+c r t)\right] L(t) } \\
& +c L(t) D,
\end{aligned}
$$

Thus ( 8$)$ is satisfied, so (a) is proved. 
(b) By (a) we have

$$
e^{(f(U)+c D) t} h(U) \mathbf{P}=e^{\int_{0}^{t} f(U+c r s) d s} h(U+c r t) e^{c D t} \mathbf{P} .
$$

Let $H(t)=e^{c D t}$ and

$$
L(t)=e^{c r t+c^{2} r t^{2} / 2+c U t} .
$$

Then $H(t) \mathbf{P}$ is uniquely determined by the conditions

$$
c D H(t) \mathbf{P}=H_{t}(t) \mathbf{P}, \quad H(0) \mathbf{P}=\mathbf{P} .
$$

On the other hand, we have (using Corollary 2.4(b))

$$
\begin{aligned}
c D L(t) \mathbf{P} & =\left(c r L_{U}(t)+c(U+r) L(t)\right) \mathbf{P} \\
& =\left(c^{2} r t+c(U+r)\right) L(t) \mathbf{P}, \\
L_{t}(t) \mathbf{P} & =\left(c r+c^{2} r t+c U\right) L(t) \mathbf{P} \\
& =c D L(t) \mathbf{P} .
\end{aligned}
$$

Since clearly $L(0) \mathbf{P}=\mathbf{P}$, it follows that $H(t) \mathbf{P}=L(t) \mathbf{P}$. Hence (6) follows from (5).

Remark on Young's lattice. In the case of Young's lattice $Y$ we can reformulate Theorem 2.5(a) in a more concrete form. We follow the notation of [M] throughout. Let $\hat{\Lambda}_{K}$ denote the ring of symmetric formal power series in variables $x=\left(x_{1}, x_{2}, \ldots\right)$ with coefficients in $K$. Define a continuous vector space isomorphism $\phi: \widehat{K} Y \rightarrow \widehat{\Lambda}_{K}$ by $\phi(\lambda)=s_{\lambda}$ for $\lambda \in Y$, where $s_{\lambda}$ denotes a Schur function. By Pieri's formula [M, (5.16)] and by [M, Example I.5.3(b)], the following diagrams commute:

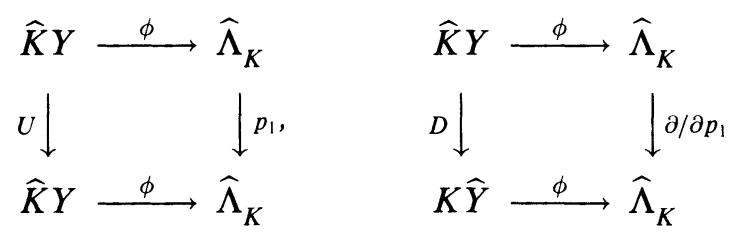

Here $p_{1}$ denotes multiplication by the power-sum $p_{1}=\sum x_{i}$, while $\partial / \partial p_{1}$ denotes partial differentiation with respect to $p_{1}$ when $f \in \widehat{\Lambda}_{K}$ is regarded as a function of $p_{1}, p_{2}, \ldots$. Thus Theorem 2.5(a) in the case $h=1$ takes the form

$$
e^{\left(f\left(p_{1}\right)+c \partial / \partial p_{1}\right) t}=e^{\int_{0}^{t} f\left(p_{1}+c s\right) d s} e^{c t \partial / \partial p_{1}} .
$$

Here $f\left(p_{1}\right)$ can be any symmetric function, but we regard it only as a function of $p_{1}$ (regard $p_{2}, p_{3}, \ldots$ as constants). Now apply (10) to a symmetric function $h\left(p_{1}\right)$. We have

$$
e^{c t \partial / \partial p_{1}} h\left(p_{1}\right)=\sum_{n \geq 0} \frac{\partial^{n} h\left(p_{1}\right)}{\partial p_{1}^{n}} c^{n} \frac{t^{n}}{n !}=h\left(p_{1}+c t\right),
$$


by Taylor's formula. Hence

$$
e^{\left(f\left(p_{1}\right)+c \partial / \partial p_{1}\right) t} h\left(p_{1}\right)=e^{\int_{0}^{t} f\left(p_{1}+c s\right) d s} h\left(p_{1}+c t\right)
$$

This formula can also be gleaned by keeping $h$ arbitrary in Theorem 2.5 (a) and letting both sides act on $\widehat{O}$. Under the isomorphism $\phi$, we have

$$
\phi\left(e^{c D t} \widehat{O}\right)=\phi\left(e^{c t \partial / \partial p_{1}} 1\right)=1 \text {, }
$$

so we obtain (11) once again.

The following special cases of Theorem 2.5 will be of the greatest use to us.

2.6. Corollary. Let $P$ be an $r$-differential poset. Then

(a) $e^{(U+D) t}=e^{r t^{2} / 2+U t} e^{D t}$,

(b) $e^{D t} e^{U t}=e^{r t^{2}+U t} e^{D t}$,

(c) $e^{D t} \mathbf{P}=e^{r t+r t^{2} / 2+U t} \mathbf{P}$,

(d) $e^{(U+D) t} \mathbf{P}=e^{r t+r t^{2}+2 U t} \mathbf{P}$,

(e) $e^{D t} e^{U t} \mathbf{P}=e^{r t+3 r t^{2} / 2+2 U t} \mathbf{P}$.

Proof. (a) Put $f(U)=U, h(U)=1$, and $c=1$ in Theorem 2.5(a).

(b) Put $f(U)=0, h(U)=e^{U t}$, and $c=1$ in Theorem 2.5(a).

(c) Put $f(U)=0, h(U)=1$, and $c=1$ in Theorem 2.5(b).

(d) Put $f(U)=U, h(U)=1$, and $c=1$ in Theorem 2.5(b).

(e) Put $f(U)=0, h(U)=e^{U t}$, and $c=1$ in Theorem 2.5(b).

I am grateful to D. Freedman for pointing out to me that Corollary 2.6(a), (b) is an immediate consequence of [G, equation (39)] (and the method of proof there is essentially the same as ours). In fact, Theorem 2.5(a) itself is well known, at least implicitly, to Lie theorists; see, e.g., [Mi, pp . 18-19].

\section{ENUMERATIVE PROPERTIES OF DIFFERENTIAL POSETS}

To convey the flavor of what we will do here, we first give a special case of subsequent much more general results.

3.1. Proposition. Let $P$ be an r-differential poset. Let $\alpha(0 \rightarrow n)$ denote the number of chains $\widehat{O}=x_{0}<x_{1}<\cdots<x_{n}$ in $P$ such that $x_{i}$ covers $x_{i-1}$ for $1 \leq i \leq n$ (so $\left.\rho\left(x_{i}\right)=i\right)$. Let $\delta_{n}$ denote the number of sequences $\hat{O}=$ $x_{0}, x_{1}, \ldots, x_{n}$ such that for $1 \leq i \leq n$, either $x_{i}$ covers $x_{i-1}$ or $x_{i-1}$ covers $x_{i}$. Then

$$
\begin{gathered}
\sum_{n \geq 0} \alpha(0 \rightarrow n) \frac{t^{n}}{n !}=\exp \left(r t+\frac{1}{2} r t^{2}\right) \\
\sum_{n \geq 0} \delta_{n} \frac{t^{n}}{n !}=\exp \left(r t+r t^{2}\right)
\end{gathered}
$$


Proof. Clearly

$$
\alpha(0 \rightarrow n)=\left\langle D^{n} \mathbf{P}, \widehat{O}\right\rangle, \quad \delta_{n}=\left\langle(U+D)^{n} \mathbf{P}, \widehat{O}\right\rangle .
$$

By Corollary 2.6(c),

$$
e^{D t} \mathbf{P}=\exp \left(r t+\frac{1}{2} r t^{2}+U t\right) \mathbf{P} .
$$

Now for any function $E(U)$ we have

$$
\langle E(U) \mathbf{P}, \widehat{O}\rangle=E(0),
$$

since $\left\langle U^{k} x, \widehat{O}\right\rangle=0$ for all $k>0$ and $x \in P$.

Thus

$$
\sum_{n \geq 0} \alpha(0 \rightarrow n) \frac{t^{n}}{n !}=\sum_{n \geq 0}\left\langle D^{n} \mathbf{P}, \widehat{O}\right\rangle \frac{t^{n}}{n !}=\left\langle e^{D t} \mathbf{P}, \widehat{O}\right\rangle=\exp \left(r t+\frac{1}{2} r t^{2}\right),
$$

as desired.

Similarly, apply (14) to Corollary $2.6(\mathrm{~d})$ to get (13).

Note. Equation (12) is well known for Young's lattice; it asserts that $\alpha(0 \rightarrow n)$ is the number of involutions in the symmetric group $S_{n}$ (see [M, Example I.5.12; St2, Proposition 17.3]). Equation (13) appears to be new even for Young's lattice, though it is easy to give a combinatorial proof based on a version of Schensted's correspondence due to this writer and G. Viennot (independently), and discussed in [Su, Lemma 8.3].

Now let $P$ be any locally finite poset, and let $x, y \in P$. A Hasse walk of length $n$ from $x$ to $y$ is a sequence $x=x_{0}, x_{1}, \ldots, x_{n}=y$ of elements of $P$ such that for $1 \leq i \leq n$ either $x_{i}$ covers $x_{i-1}$ or $x_{i}$ is covered by $x_{i-1}$. Thus a Hasse walk is just a walk (in the usual graph-theoretical sense, e.g., [B-C-L, p. 20; B-M, p . 12]) on the Hasse diagram (considered as a graph) of $P$. If $S, T$ are subsets of $P$, we also say that the Hasse walk $x_{0}, x_{1}, \ldots, x_{n}$ goes from $S$ to $T$ if $x_{0} \in S$ and $x_{n} \in T$. Many of our enumerative results concerning differential posets will deal with counting Hasse walks satisfying various conditions. In this regard, it will be convenient to generalize the notation $\alpha(0 \rightarrow n)$ in a way best explained by an example. Namely, $\alpha(3 \rightarrow 7 \rightarrow 5 \rightarrow 8)$ denotes the number of Hasse walks $x_{0}, x_{1}, \ldots, x_{9}$ in $P$ such that

$$
\begin{gathered}
x_{0}<x_{1}<x_{2}<x_{3}<x_{4}, \quad \text { of ranks } 3,4,5,6,7, \\
x_{4}>x_{5}>x_{6}, \quad \text { of ranks } 7,6,5, \\
x_{6}<x_{7}<x_{8}<x_{9}, \quad \text { of ranks } 5,6,7,8 .
\end{gathered}
$$

Thus if we let $e(x)$ denote the number of maximal chains in the interval $[\hat{O}, x]$, then

$$
\alpha(0 \rightarrow n \rightarrow 0)=\sum_{x \in P_{n}} e(x)^{2}
$$


while

$$
\begin{aligned}
\alpha(n \rightarrow 0 \rightarrow n) & =\left(\sum_{x \in P_{n}} e(x)\right)^{2} \\
& =\alpha(n \rightarrow 0)^{2}=\alpha(0 \rightarrow n)^{2}
\end{aligned}
$$

We also let

$$
F(P, q)=\sum_{x \in P} q^{\rho(x)}=\sum_{n \geq 0}\left(\# P_{n}\right) q^{n}=\sum_{n \geq 0} \alpha(n) q^{n},
$$

the rank-generating function of $P$ [St6, p. 99]. For the lattice $Y^{r}$,

$$
F\left(Y^{r}, q\right)=\prod_{i \geq 1}\left(1-q^{i}\right)^{-r}
$$

3.2. Theorem. Let $P$ be an $r$-differential poset, and define $A_{k}(q)$ by

$$
\sum_{n \geq 0} \alpha(n \rightarrow n+k) q^{n}=A_{k}(q) F(P, q) .
$$

Then

$$
\sum_{k \geq 0} A_{k}(q) \frac{t^{k}}{k !}=\exp \left(\frac{r t}{1-q}+\frac{r t^{2}}{2\left(1-q^{2}\right)}\right) .
$$

In particular, $A_{k}(q)$ is a rational function of $q$ whose only poles are at +1 (for $k \geq 1)$ and -1 (for $k \geq 2)$.

Proof. Write

$$
G(q, t)=F(P, q) \sum_{k \geq 0} A_{k}(q) \frac{t^{k}}{k !}=\sum_{n \geq 0} \sum_{k \geq 0} \alpha(n \rightarrow n+k) \frac{q^{n} t^{k}}{k !} .
$$

Let $\gamma: \widehat{K} P \rightarrow K[[q]]$ be the continuous linear transformation defined by $\gamma(x)=$ $q^{\rho(x)}$ for $x \in P$. (Thus in particular $\gamma(\mathbf{P})=F(P, q)$.)

Now

$$
\begin{aligned}
\gamma\left(e^{D t} \mathbf{P}\right) & =\sum_{k \geq 0} \frac{\gamma\left(D^{k} \mathbf{P}\right) t^{k}}{k !} \\
& =\sum_{n \geq 0} \sum_{k \geq 0} \frac{\alpha(n \rightarrow n+k) q^{n} t^{k}}{k !}=G(q, t) .
\end{aligned}
$$

On the other hand, by Corollary 2.6(c) we have

$$
\begin{aligned}
\gamma\left(e^{D t} \mathbf{P}\right) & =e^{r t+r t^{2} / 2} \gamma\left(e^{U t} \mathbf{P}\right) \\
& =e^{r t+r t^{2} / 2} \sum_{n \geq 0} \sum_{k \geq 0} \frac{\alpha(n-k \rightarrow n) q^{n} t^{k}}{k !} \\
& =e^{r t+r t^{2} / 2} \sum_{n \geq 0} \sum_{k \geq 0} \frac{\alpha(n-k \rightarrow n) q^{n-k}(q t)^{k}}{k !} \\
& =e^{r t+r t^{2} / 2} G(q, q t) .
\end{aligned}
$$


Thus $G(q, t)$ satisfies

$$
G(q, t)=e^{r t+r t^{2} / 2} G(q, q t), \quad G(q, 0)=F(P, q),
$$

and these conditions determine $G(q, t)$ uniquely. But the power series

$$
F(P, q) \exp \left(\frac{r t}{1-q}+\frac{r t^{2}}{2\left(1-q^{2}\right)}\right)
$$

is easily seen to satisfy (20), so the theorem is proved.

The rational functions $A_{k}(q)$ for $0 \leq k \leq 5$ are given by

$$
\begin{aligned}
& A_{0}(q)=1 \\
& A_{1}(q)=\frac{r}{1-q}, \\
& A_{2}(q)=\frac{r(r+1)+r(r-1) q}{(1-q)\left(1-q^{2}\right)}, \\
& A_{3}(q)=\frac{r^{2}(r+3)+r^{2}(r-3) q}{(1-q)^{2}\left(1-q^{2}\right)}, \\
& A_{4}(q)=\frac{r^{2}\left(r^{2}+6 r+3\right)+2 r^{2}\left(r^{2}-3\right) q+r^{2}\left(r^{2}-6 r+3\right) q^{2}}{(1-q)^{2}\left(1-q^{2}\right)^{2}}, \\
& A_{5}(q)=\frac{r^{3}\left(r^{2}+10 r+15\right)+2 r^{3}\left(r^{2}-15\right) q+r^{3}\left(r^{2}-10 r+15\right) q^{2}}{(1-q)^{3}\left(1-q^{2}\right)^{2}} .
\end{aligned}
$$

Note that for any locally finite graded poset with $\widehat{O}$ and with finite rank sizes, (16) holds when $k=0$. Moreover, it is easy to see that properties (D1) and (D3) alone imply (16) when $k=1$. However, (D1) and (D3) are not sufficient to imply (16) when $k \geq 2$.

In the case $P=Y$ (Young's lattice), the problem of evaluating $A_{k}(q)$ was raised in [St6, solution to Exercise 4.21(b)] (where it was denoted $A_{[k]}(q)$ ). Theorem 3.2 can also be proved for $P=Y$ by proving the symmetric function identity

$$
\sum_{\lambda, \mu} s_{\lambda / \mu}(x) q^{|\mu|}=F(Y, q) \prod_{n \geq 0}\left[\prod_{i<j}\left(1-q^{n} x_{i} x_{j}\right)^{-1} \prod_{i}\left(1-q^{n} x_{i}\right)^{-1}\right]
$$

and then considering the coefficient of $x_{1} x_{2} \cdots x_{k}$ on both sides. A combinatorial proof of (21) appears in [S-S].

We now come to one of our main results on enumerative properties of differential posets.

3.3. Theorem. Let $P$ be an r-differential poset. Let $f(U, D)$ be any noncommutative power series in the variables $U$ and $D$ over the field $K$ such that $f(U, D)$ defines a linear transformation $\widehat{K} P \rightarrow \widehat{K} P$. Then

$$
\gamma(f(U, D) \mathbf{P})=A_{f}(q) F(P, q)
$$


for some power series $A_{f}(q)$ depending only on $f$ and $r$ (not on $P$ ). To compute $A_{f}(q)$ explicitly, repeatedly use Theorem 2.3 and Corollary 2.4(b) to write

$$
f(U, D) \mathbf{P}=g(U) \mathbf{P} .
$$

Then in the power series expansion of $g(U)$, replace $U^{k}$ by $q^{k} A_{k}(q)$ to get $A_{f}(q)$.

Proof. It is clear that repeated applications of Theorem 2.3 and Corollary 2.4(b) allow us to write $f(U, D) \mathbf{P}=g(U) \mathbf{P}$ for some $g(U)$. Now

$$
\sum_{n \geq 0} \alpha(n \rightarrow n+k) q^{n+k}=\gamma\left(U^{k} \mathbf{P}\right),
$$

so by (16) we have

$$
\gamma\left(U^{k} \mathbf{P}\right)=q^{k} A_{k}(q) F(P, q) .
$$

Since $\gamma$ is linear and continuous, the proof follows.

Let us consider further the polynomial $g(U)$ of $(23)$ when $f(U, D)$ consists of a single word $w \in\{U, D\}^{*}$, where $\{U, D\}^{*}$ denotes the free monoid with generators $U$ and $D$. (Every $f(U, D)$ is of course an infinite linear combination of such $w$.) For any $w \in\{U, D\}^{*}$ define inductively a polynomial $g_{w}(z)$ as follows:

$$
\begin{aligned}
g_{\phi}(z) & =1, \quad \text { where } \phi \text { denotes the empty word, } \\
g_{U v}(z) & =z g_{v}(z), \quad \text { for any } v \in\{U, D\}^{*}, \\
g_{D v}(z) & =r g_{v}^{\prime}(z)+(r+z) g_{v}(z), \quad \text { for any } v \in\{U, D\}^{*},
\end{aligned}
$$

where ' denotes differentiation. Equivalently, for $w=w(U, D)$ we have

$$
g_{u}(z)=e^{-z-z^{2} / 2 r} w(z, r d / d z) e^{z+z^{2} / 2 r},
$$

since it is easy to check that the right-hand side of (25) satisfies the conditions (24).

3.4. Corollary. Let $P$ be an $r$-differential poset. If $w \in\{U, D\}^{*}$, then

$$
\gamma(w \mathbf{P})=A_{w}(q) F(P, q),
$$

where $A_{w}(q)$ is obtained from $g_{w}(z)$ by replacing $z^{k}$ with $q^{k} A_{k}(q)$. (Thus $A_{w}(q)$ is a nonnegative integral linear combination of the rational functions $q^{k} A_{k}(q)$.)

Proof. We claim that

$$
w \mathbf{P}=g_{w}(U) \mathbf{P},
$$

from which the proof will follow from Theorem 3.3. We use induction on the length of $w$. If $w=\phi$, then (26) is clear since $\phi \mathbf{P}=\mathbf{P}$. Now assuming (26) 
for all words of length $l-1$, let $v$ have length $l-1$. Then

$$
\begin{aligned}
U v \mathbf{P} & =U g_{v}(U) \mathbf{P}=g_{U v}(U) \mathbf{P}, \\
D v \mathbf{P} & =D g_{v}(U) \mathbf{P} \\
& =\left[r g_{v}^{\prime}(U)+(r+U) g_{v}(U)\right] \mathbf{P} \quad \text { (by Corollary 2.4(b)) } \\
& =g_{D v}(U) \mathbf{P},
\end{aligned}
$$

so the proof follows.

3.5. Example. Let us illustrate the meaning of Corollary 3.4 with the example $w=U D U U$. Thus

$$
\begin{aligned}
\gamma(U D U U \mathbf{P}) & =\sum_{n \geq 0} \alpha(n-2 \rightarrow n \rightarrow n-1 \rightarrow n) q^{n} \\
& =\sum_{n \geq 0} \alpha(n \rightarrow n-1 \rightarrow n \rightarrow n-2) q^{n}
\end{aligned}
$$

We have

$$
\begin{aligned}
g_{\phi}(z) & =1 \\
g_{U}(z) & =z \\
g_{U U}(z) & =z^{2} \\
g_{D U U}(z) & =2 r z+(r+z) z^{2} \\
& =2 r z+r z^{2}+z^{3} \\
g_{U D U U}(z) & =2 r z^{2}+r z^{3}+z^{4} .
\end{aligned}
$$

Hence

$$
A_{U D U U}(q)=2 r q^{2} A_{2}(q)+r q^{3} A_{3}(q)+q^{4} A_{4}(q) .
$$

It follows that the coefficient of $q^{n}$ in

$$
F(P, q)\left(2 r q^{2} A_{2}(q)+r q^{3} A_{3}(q)+q^{4} A_{4}(q)\right)
$$

is equal to the number of Hasse walks $x_{0}, x_{1}, x_{2}, x_{3}, x_{4}$ in $P$ of the form $x_{0}<x_{1}<x_{2}>x_{3}<x_{4}$, where $\rho\left(x_{4}\right)=n$.

Theorem 3.3 asserts that certain combinatorial invariants of $P$ depend only on $F(P, q)$ (and of course on $r$ ). There is a special case which is even independent of $F(P, q)$.

3.6. Corollary. Preserve the notation of Theorem 3.3. Then $\langle f(U, D) \mathbf{P}, \widehat{O}\rangle=$ $A_{f}(0)$.

Proof. By definition of $\gamma$, the coefficient of $q^{n}$ in $\gamma(f(U, D) \mathbf{P})$ is equal to

$$
\sum_{\rho(x)=n}\langle f(U, D) \mathbf{P}, x\rangle .
$$

Now take the constant term of both sides of (22). The left-hand side becomes $\langle f(U, D) \mathbf{P}, \widehat{O}\rangle$, while the right-hand side becomes $A_{f}(0)$. 
Let us now consider the special case of Corollary 3.6 where $f(U, D)$ is a single word $w \in\{U, D\}^{*}$. Surprisingly there is a simple formula for $A_{w}(0)$. More generally, we can give a formula for $\langle w \widehat{O}, x\rangle$ for any $x \in P$. Let us call the word $w=w(U, D)=w_{1} w_{2} \cdots w_{l}$ of length $l$ a valid $x$-word if $\langle w \widehat{O}, x\rangle \neq 0$. Equivalently, (a) for all $1 \leq i \leq l$, the number of $D$ 's among $w_{i}, w_{i+1}, \ldots, w_{l}$ does not exceed the number of $U$ 's, and (b) the difference between the number of $U$ 's and number of $D$ 's in $w$ is the rank $\rho(x)$ of $x$.

3.7. Theorem. Let $P$ be an $r$-differential poset, and let $x \in P$. Suppose that $w=w_{1} w_{2} \cdots w_{1}$ is a valid $x$-word. Let $S=\left\{i: w_{i}=D\right\}$. For each $i \in S$, let $a_{i}$ be the number of $D$ 's in $w$ to the right of $w_{i}$, and let $b_{i}$ be the number of $U$ 's in $w$ to the right of $w_{i}$. Set $d_{i}=b_{i}-a_{i}$. Then

$$
\langle w \widehat{O}, x\rangle=e(x) r^{\# S} \prod_{i \in S} d_{i},
$$

where $e(x)$ is as in (15). Thus (summing over all $x \in P_{n}$, where $n$ is the rank of elements $x$ for which $w$ is a valid $x$-word),

$$
\langle w \widehat{O}, \mathbf{P}\rangle=\alpha(0 \rightarrow n) r^{\# S} \prod_{i \in S} d_{i} .
$$

Proof. By successive uses of Theorem 2.2(b) we can put $w$ in the form

$$
w=\sum_{i, j} c_{i j}(w) U^{i} D^{j},
$$

where $c_{i j}(w)$ is a polynomial in $r$, and where if $c_{i j}(w) \neq 0$ then $i-j=\rho(x)$. Moreover, this representation is easily seen to be unique. Now

$$
U w=\sum_{i, j} c_{i j}(w) U^{i+1} D^{j} \Rightarrow c_{i j}(U w)=c_{i-1, j}(w) .
$$

Moreover, by (4) we have

$$
\begin{aligned}
D w & =\sum_{i, j} c_{i j}(w) D U^{i} D^{j} \\
& =\sum_{i, j} c_{i j}(w)\left(U^{i} D+i r U^{i-1}\right) D^{j} \\
& \Rightarrow c_{i j}(D w)=c_{i, j-1}(w)+(i+1) r c_{i+1, j}(w) .
\end{aligned}
$$

In particular, when $j=0$ we have

$$
\begin{gathered}
c_{i 0}(U w)=c_{i-1,0}(w), \\
c_{i 0}(D w)=(i+1) r c_{i+1,0}(w) .
\end{gathered}
$$

Now let (28) operate on $\widehat{O}$. Since $D^{j} \widehat{O}=0$ for $j>0$, we get (setting $\rho=\rho(x)), w \widehat{O}=c_{\rho 0}(w) U^{\rho} \widehat{O}$. Thus

$$
\langle w \widehat{O}, x\rangle=c_{\rho 0}(w)\left\langle U^{\rho} \widehat{O}, x\right\rangle=c_{\rho 0}(w) e(x) .
$$


It is easy to see from $(29(a),(b))$ that

$$
c_{\rho 0}(w)=r^{\# S} \prod_{i \in S} d_{i},
$$

and the proof follows.

3.8. Example. Let us continue Example 3.5 by asking for the value of $\langle U D U U \widehat{O}, x\rangle=\langle\widehat{O}, D D U D x\rangle$ for $x \in P_{2}$, i.e., the number of Hasse walks $\widehat{O}=x_{0}<x_{1}<x_{2}>x_{3}<x_{4}=x$. In Theorem 3.7 take $w=U D U U$, so $S=\{2\}, a_{2}=2, b_{2}=0, d_{2}=2$. We get $\langle U D U U \hat{O}, x\rangle=2 r e(x)$. Thus also

$$
\langle U D U U \widehat{O}, \mathbf{P}\rangle=2 r \alpha(0 \rightarrow 2)=2 r^{2}(r+1),
$$

since $\alpha(0 \rightarrow 2)=r(r+1)$ by (12). We obtain the same answer by dividing (27) by $q^{2}$ and putting $q=0$.

A special case of Theorem 3.7 deserves special mention because it is well known for Young's lattice [St2, Proposition 17.2].

3.9. Corollary. Let $P$ be an r-differential poset. Then, using the notation of (15),

$$
\alpha(0 \rightarrow n \rightarrow 0)=\sum_{x \in P_{n}} e(x)^{2}=r^{n} n ! .
$$

Proof. This is just the case $w=D^{n} U^{n}$ (and thus $x=\widehat{O}$ ) of Theorem 3.7.

The next result evaluates the rational functions $A_{w}(q)$ of Corollary 3.4 in terms of generating functions. Let us introduce the following notation. Let $\Omega$ denote the set of all infinite sequences $\nu=\left(\nu_{1}, \nu_{2}, \ldots\right)$ of nonnegative integers such that $\sum \nu_{i}<\infty$. Write $t^{\nu}=t_{1}^{\nu_{1}} t_{2}^{\nu_{2}} \cdots$, and similarly for $t^{\mu}, s^{\nu}, s^{\mu}$, etc. Define $\nu !=\nu_{1} ! \nu_{2} ! \cdots$, and set $S=\sum s_{i}$ and $T=\sum t_{i}$. Finally write $A(w, q)$ instead of $A_{w}(q)$.

3.10. Proposition. Let $P$ be an r-differential poset. Then

$$
\begin{aligned}
\sum_{\mu, \nu \in \Omega} A\left(\cdots D^{\mu_{2}} U^{\nu_{2}} D^{\mu_{1}} U^{\nu_{1}}, q\right) \frac{s^{\mu} t^{\nu}}{\mu ! \nu !} & \\
=\exp r\left[\frac{q(S+T)}{1-q}+\frac{q^{2}(S+T)^{2}}{2\left(1-q^{2}\right)}\right. & +\sum_{i<j} s_{i} s_{j} \\
& \left.+\sum_{i \geq j} s_{i} t_{j}+S+\frac{1}{2} \sum_{i} s_{i}^{2}\right] .
\end{aligned}
$$

Proof. We first prove by induction on $k$ that

$$
\begin{aligned}
G_{k} \mathbf{P} & =G_{k}\left(U, D, s_{1}, \ldots, s_{k}, t_{1}, \ldots, t_{k}\right) \mathbf{P} \\
& :=e^{D s_{k}} e^{U t_{k}} \cdots e^{D s_{1}} e^{U t_{1}} \mathbf{P} \\
& =\exp \left[r E_{k}+U\left(S_{k}+T_{k}\right)\right] \mathbf{P}
\end{aligned}
$$


where

$$
\begin{gathered}
E_{k}=\sum_{1 \leq i<j \leq k} s_{i} s_{j}+\sum_{k \geq i \geq j \geq 1} s_{i} t_{j}+\sum_{i=1}^{k}\left(s_{i}+s_{i}^{2} / 2\right), \\
S_{k}=\sum_{i=1}^{k} s_{i}, \quad T_{k}=\sum_{i=1}^{k} t_{i} .
\end{gathered}
$$

Equation (30) is clear for $k=0$, so assume it for $k-1$. Then

$$
\begin{aligned}
G_{k} \mathbf{P} & =e^{D s_{k}} e^{U t_{k}} G_{k-1} \mathbf{P} \\
& =e^{D s_{k}} e^{U t_{k}} \exp \left[r E_{k-1}+U\left(S_{k-1}+T_{k-1}\right)\right] \mathbf{P}
\end{aligned}
$$

Now apply Theorem 2.5(b) to the case $f(U)=0, c=1, t=s_{k}$, and

$$
h(U)=e^{U t_{k}} \exp \left[r E_{k-1}+U\left(S_{k-1}+T_{k-1}\right)\right] .
$$

We obtain

$$
G_{k} \mathbf{P}=h\left(U+r s_{k}\right) \exp \left(r s_{k}+\frac{1}{2} r s_{k}^{2}+s_{k} U\right) \mathbf{P} .
$$

It is easy to check that the right-hand sides of (30) and (31) agree, so (30) is proved.

Now apply the linear transformation $\gamma$ defined in the proof of Theorem 3.2 to $(30)$. We get

$$
\begin{aligned}
F(P, q) & \sum_{\mu, \nu \in \mathbb{N}^{k}} A\left(D^{\mu_{k}} U^{\nu_{k}} \ldots D^{\mu_{1}} U^{\nu_{1}}, q\right) \frac{s^{\mu} t^{\nu}}{\mu ! \nu !} \\
& =e^{r E_{k}} \gamma\left(e^{U\left(S_{k}+T_{k}\right)} \mathbf{P}\right) \\
& =F(P, q) \exp r\left[E_{k}+\frac{q\left(S_{k}+T_{k}\right)}{1-q}+\frac{q^{2}\left(S_{k}+T_{k}\right)^{2}}{2\left(1-q^{2}\right)}\right],
\end{aligned}
$$

by (19) and Theorem 3.2. Now let $k \rightarrow \infty$ in (32) and divide by $F(P, q)$ to complete the proof.

We next consider a variation of our previous results which involves closed Hasse walks, i.e., Hasse walks which begin and end at the same element of $P$. We use the notation $\kappa$ instead of $\alpha$ to denote that we are enumerating closed walks. Thus, e.g., $\kappa(n \rightarrow n+k \rightarrow n)$ denotes the number of Hasse walks $x_{0}, x_{1}, \ldots, x_{2 k}$ such that $\rho\left(x_{0}\right)=n, x_{0}<x_{1}<\cdots<x_{k}>x_{k+1}>\cdots>x_{2 k}$, and $x_{2 k}=x_{0}$. If $e(x, y)$ denotes the number of maximal chains of the interval $[x, y]$ (with $e(x, y)=0$ if $x \not z y$ ), then it follows that

$$
\kappa(n \rightarrow n+k \rightarrow n)=\sum_{x \in P_{n}} \sum_{y \in P_{n+k}} e(x, y)^{2} .
$$

We come to the "closed analogue" of Theorem 3.2. 
3.11. Theorem. Let $P$ be an $r$-differential poset, and fix $k \in \mathbb{N}$. Then

$$
\sum_{n \geq 0} \kappa(n \rightarrow n+k \rightarrow n) q^{n}=r^{k} k !(1-q)^{-k} F(P, q) .
$$

Proof. We have

$$
\kappa(n \rightarrow n+k \rightarrow n)=\sum_{x \in P_{n}}\left\langle D^{k} U^{k} x, x\right\rangle .
$$

Since $\left\langle D^{i} U^{j} x, x\right\rangle=0$ if $i \neq j$, it follows that

$$
\begin{aligned}
G(q, t) & :=\sum_{k, n \geq 0} \kappa(n \rightarrow n+k \rightarrow n) \frac{t^{k} q^{n}}{(k !)^{2}} \\
& =\sum_{x \in P}\left\langle e^{D t} e^{U} x, x\right\rangle q^{\rho(x)} \\
& =e^{r t} \sum_{x \in P}\left\langle e^{U} e^{D t} x, x\right\rangle q^{\rho(x)},
\end{aligned}
$$

by Theorem 2.5(a). But

$$
\kappa(n-k \rightarrow n \rightarrow n-k)=\kappa(n \rightarrow n-k \rightarrow n)=\sum_{x \in P_{n}}\left\langle U^{k} D^{k} x, x\right\rangle .
$$

Hence

$$
\begin{aligned}
G(q, t) & =e^{r t} \sum_{k, n \geq 0} \kappa(n-k \rightarrow n \rightarrow n-k) \frac{t^{k} q^{n}}{(k !)^{2}} \\
& =e^{r t} G(q, q t) .
\end{aligned}
$$

The unique solution to $G(q, t)=e^{r t} G(q, q t)$ satisfying $G(q, 0)=F(P, q)$ is given by

$$
G(q, t)=F(P, q) \exp \left(\frac{r t}{1-q}\right) .
$$

Equating coefficients of $t^{k} /(k !)^{2}$ on both sides of (36) completes the proof. $n$

Theorem 3.11 is yet another result already known for Young's lattice. It was conjectured by B. Sagan and then proved combinatorially in [S-S]. It can also be proved by equating coefficients of $x_{1} \cdots x_{k} y_{1} \cdots y_{k} q^{n}$ on both sides of the identity

$$
\sum_{\lambda, \mu} s_{\lambda / \mu}(x) s_{\lambda / \mu}(y) q^{|\mu|}=\prod_{m \geq 1}\left(1-q^{m}\right)^{-1} \prod_{i, j}\left(1-x_{i} y_{j} q^{m-1}\right)^{-1} .
$$

This identity is essentially the special case $\left(v_{1}, v_{2}, v_{3}, \ldots\right)=(q, 0,0, \ldots)$ of [St4, Theorem 5.1].

The following theorem is the "closed analogue" of Theorem 3.3. 
3.12. Theorem. Let $P$ be an r-differential poset, and let $f(U, D)$ be as in Theorem 3.3. Then

$$
\sum_{x \in P}\langle f(U, D) x, x\rangle q^{\rho(x)}=B_{f}(q) F(P, q)
$$

for some power series $B_{f}(q)$ depending only on $f$ and $r$ (not on $P$ ). To compute $B_{f}(q)$ explicitly, repeatedly use Corollary $2.4(\mathrm{a})$ to write

$$
f(U, D)=\sum_{i, j} a_{i j} U^{i} D^{j}
$$

Then

$$
B_{f}(q)=\sum_{k} k ! a_{k k} r^{k} q^{k}(1-q)^{-k}
$$

Proof. Clearly Corollary 2.4(a) allows $f(U, D)$ to be written in the form (37). The proof follows from the fact that $\left\langle U^{i} D^{j} x, x\right\rangle=0$ if $i \neq j$, together with (35) and Theorem 3.11.

3.13. Example. Let $\kappa(n \rightarrow n+1 \rightarrow n-1 \rightarrow n)$ denote the number of closed Hasse walks $x_{0}<x_{1}>x_{2}>x_{3}<x_{4}=x_{0}$ with $\rho\left(x_{0}\right)=n$. Thus

$$
\sum_{n \geq 0} \kappa(n \rightarrow n+1 \rightarrow n-1 \rightarrow n) q^{n}=\sum_{x \in P}\langle U D D U x, x\rangle q^{\rho(x)} .
$$

It is easily computed that $U D D U=U^{2} D^{2}+2 r U D$. Hence by Theorem 3.12,

$$
\sum_{n \geq 0} \kappa(n \rightarrow n+1 \rightarrow n-1 \rightarrow n) q^{n}=\left(\frac{2 q r^{2}}{1-q}+\frac{2 q^{2} r^{2}}{(1-q)^{2}}\right) F(P, q) .
$$

If, as in the previous example, $f(U, D)$ is a single word $w(U, D) \in$ $\{U, D\}^{*}$ which contains $m U$ 's and $m D$ 's, then it is easy to see that

$$
w(U, D)=\sum_{k} a_{k} U^{k} D^{k},
$$

where

$$
\sum a_{k} z^{k}=e^{-z / r} r^{m} w\left(z, \frac{d}{d z}\right) e^{z / r} .
$$

A corollary of Theorem 3.12 is of particular interest.

3.14. Corollary. Let $P$ be an $r$-differential poset. Let $\kappa_{2 k}(n)$ denote the total number of closed Hasse walks of length $2 k$ starting at $P_{n}$. Then for fixed $k$,

$$
\sum_{n \geq 0} \kappa_{2 k}(n) q^{n}=\frac{(2 k) ! r^{k}}{2^{k} k !}\left(\frac{1+q}{1-q}\right)^{k} F(P, q) .
$$


Proof. We have

$$
\begin{aligned}
\sum_{k, n \geq 0} \kappa_{2 k}(n) \frac{q^{n} t^{2 k}}{(2 k) !} & =\sum_{x \in P}\left\langle e^{(D+U) t} x, x\right\rangle q^{\rho(x)} \\
& =e^{r t^{2} / 2} \sum_{x \in P}\left\langle e^{U t} e^{D t} x, x\right\rangle q^{\rho(x)}
\end{aligned}
$$

(by Corollary 2.6(a))

$$
=e^{r t^{2} / 2} G\left(q, q t^{2}\right),
$$

where $G(q, t)$ is defined by (34). Thus by (36),

$$
\sum_{k, n \geq 0} \kappa_{2 k}(n) \frac{q^{n} t^{2 k}}{(2 k) !}=F(P, q) \exp \left(\frac{1}{2} r t^{2}+\frac{r q t^{2}}{1-q}\right) \text {. }
$$

Extracting the coefficient of $t^{2 k} /(2 k)$ ! on both sides yields the desired result.

If we set $q=0$ in Corollary 3.14, we obtain

$$
\kappa_{2 k}(0)=(2 k) ! r^{k} / 2^{k} k ! \text {. }
$$

This result is already known for Young's lattice. It follows from [Be] using tools from representation theory (viz., the theory of the Brauer algebra of $\operatorname{Sp}(2 n)$ or $O(n)$ ), and was subsequently given a combinatorial proof by this writer and $\mathrm{G}$. Viennot (independently). See also [Su, Lemma 8.3].

Another generalization of (39) is the following.

3.15. Corollary. Preserve the notation of Theorem 2.5. Then

$$
\left\langle e^{(f(U)+D) t} h(U) \widehat{O}, \widehat{O}\right\rangle=e^{\int_{0}^{t} f(r s) d s} h(r t) .
$$

Proof. Apply both sides of Theorem 2.5(a) to $\widehat{O}$, and then extract the coefficient of $\widehat{O}$. Since $e^{D t} \widehat{O}=\widehat{O}$, the proof follows from (14).

The following is a sample special case of the preceding corollary.

3.16. Corollary. Let $P$ be an r-differential poset, and fix $k, n \in \mathbb{P}$. Then the number of closed Hasse walks

$$
\widehat{O}=x_{0}<x_{1}<\cdots<x_{k}, x_{k+1}, \ldots, x_{2(k+n)}=\widehat{O}
$$

in $P$ is equal to $r^{n+k}(2 n+k) ! / 2^{n} n$ !.

Proof. The desired number $N$ is given by

$$
N=\left\langle(U+D)^{2 n+k} U^{k} \widehat{O}, \widehat{O}\right\rangle,
$$

which by the preceding corollary is the coefficient of $t^{2 n+k} /(2 n+k)$ ! in

$$
e^{\int_{0}^{t} r s d s}(r t)^{k}=r^{k} t^{k} e^{r t^{2} / 2}=\sum_{n \geq 0} \frac{r^{n+k}(2 n+k) !}{2^{n} n !} \frac{t^{2 n+k}}{(2 n+k) !},
$$

so the proof follows.

We next give the analogue of Corollary 3.14 for arbitrary (i.e ., not necessarily closed) Hasse walks. 
3.17. Proposition. Let $P$ be an $r$-differential poset. Let $\delta_{n}(j \rightarrow k)$ denote the number of Hasse walks from $P_{j}$ to $P_{k}$ of length $n$. Then

$$
\sum_{j, k, n \geq 0} \delta_{n}(j \rightarrow k) \frac{q^{j} z^{k} t^{n}}{n !}=F(P, q z) \exp r\left[\frac{(q+z) t}{1-q z}+\frac{\left(1+q^{2}\right)\left(1+z^{2}\right) t^{2}}{2\left(1-q^{2} z^{2}\right)}\right] .
$$

Proof. We have

$$
\sum_{j, k, n \geq 0} \delta_{n}(j \rightarrow k) \frac{q^{j} z^{k-j} t^{n}}{n !}=\gamma\left(e^{\left(z^{-1} U+z D\right) t} \mathbf{P}\right) .
$$

Apply Theorem 2.5(b) to the case $f(U)=z^{-1} U, c=z, h(U)=1$ to get

$$
e^{\left(z^{-1} U+z D\right) t} \mathbf{P}=\exp \left[r z t+\frac{1}{2} r\left(1+z^{2}\right) t+\left(z+z^{-1}\right) U t\right] \mathbf{P} .
$$

It follows from Theorem 3.2 that

$$
\gamma\left(e^{\left(z+z^{-1}\right) U t} \mathbf{P}\right)=F(P, q) \exp \left[\frac{r q\left(z+z^{-1}\right) t}{1-q}+\frac{r q^{2}\left(z+z^{-1}\right) t^{2}}{2\left(1-q^{2}\right)}\right] .
$$

Combining (40), (41), and (42) yields

$$
\begin{aligned}
& \sum_{j, k, n \geq 0} \delta_{n}(j \rightarrow k) \frac{q^{j} z^{k-j} t^{n}}{n !} \\
& \quad=F(P, q) \exp r\left[z t+\frac{1}{2}\left(1+z^{2}\right) t^{2}+\frac{q\left(z+z^{-1}\right) t}{1-q}+\frac{q^{2}\left(z+z^{-1}\right)^{2} t^{2}}{2\left(1-q^{2}\right)}\right] \\
& \quad=F(P, q) \exp r\left[\frac{\left(q z^{-1}+z\right) t}{1-q}+\frac{\left(1+q^{2} z^{-2}\right)\left(1+z^{2}\right) t^{2}}{2\left(1-q^{2}\right)}\right] .
\end{aligned}
$$

Now substitute $q z$ for $q$ to complete the proof.

Note that setting $z=0$ in Proposition 3.17 yields (since $\delta_{n}(0 \rightarrow j)=$ $\left.\delta_{n}(j \rightarrow 0)\right)$

$$
\begin{aligned}
\sum_{j, n \geq 0} \delta_{n}(0 \rightarrow j) \frac{q^{j} t^{n}}{n !} & =\exp r\left[q t+\frac{1}{2}\left(1+q^{2}\right) t^{2}\right] \\
& =e^{r t^{2} / 2} e^{r\left(q t+q^{2} t^{2} / 2\right)} \\
& =e^{r t^{2} / 2} \sum_{j \geq 0} \alpha(0 \rightarrow j) \frac{q^{j} t^{j}}{j !}
\end{aligned}
$$

from which it follows that

$$
\delta_{j+2 m}(0 \rightarrow j)=\frac{\alpha(0 \rightarrow j) r^{m}(j+2 m) !}{j ! 2^{m} m !} .
$$

Note that if we put $j=0$ then we get (39), while putting $q=1$ in the above generating function yields (13). It can be shown, similarly to the proof 
of Theorem 3.7, that if $x \in P_{j}$ then the number $\bar{e}_{j+2 m}(x)$ of Hasse paths of length $j+2 m$ from $\widehat{O}$ to $x$ is given by

$$
\bar{e}_{j+2 m}(x)=\frac{e(x) r^{m}(j+2 m) !}{j ! 2^{m} m !} .
$$

For Young's lattice the above formula for $\bar{e}_{j+2 m}(x)$ is given a combinatorial proof in [Su, Lemma 8.7].

\section{CHARACTERISTIC POLYNOMIALS}

In this section we will compute the characteristic polynomials of certain linear transformations and matrices associated with differential posets. We will apply these results to obtain more structural properties of differential posets.

If $A: \widehat{K} P \rightarrow \widehat{K} P$ is a linear transformation, then let $A_{j}$ denote the restriction of $A$ to $K P_{j}$. Note that such notation as $A B_{j}$ is unambiguous since $A\left(B_{j}\right)$ and $(A B)_{j}$ have the same meaning. For a linear transformation $A: V \rightarrow V$ on a finite-dimensional vector space $V$, write $\mathrm{Ch} A=\mathrm{Ch}(A, \lambda)$ for the characteristic polynomial $\operatorname{det}(\lambda I-A)$ (normalized to be monic) of $A$. For a differential poset $P$, we let $p_{j}=\# P_{j}$, the number of elements of rank $j$, and we set $\Delta p_{j}=p_{j}-p_{j-1}$.

4.1. Theorem. Let $P$ be an $r$-differential poset and let $j \in \mathbb{N}$. Then

$$
\operatorname{Ch}\left(U D_{j}\right)=\prod_{i=0}^{j}(\lambda-r i)^{\Delta p_{j-i}} .
$$

Proof. Induction on $j$. Since clearly $\mathrm{Ch} U D_{0}=\lambda,(43)$ is valid for $j=0$. Assume (43) for $j$. Suppose that $A: V \rightarrow W$ and $B: W \rightarrow V$ are linear transformations on finite-dimensional vector spaces $V$ and $W$ and that $\operatorname{dim} V=v$ and $\operatorname{dim} W=w$. Then

$$
\mathrm{Ch}(B A)=\lambda^{v-u} \mathrm{Ch}(A B) .
$$

Applying this fact to $D_{j+1}$ and $U_{j}$ yields

$$
\begin{aligned}
\mathrm{Ch}\left(U D_{j+1}, \lambda\right) & =\lambda^{\Delta p_{j+1}} \operatorname{Ch}\left(D U_{j}, \lambda\right) \\
& =\lambda^{\Delta p_{j+1}} \operatorname{Ch}\left(U D_{j}+r I, \lambda\right) \quad(\text { by Theorem 2.2) } \\
& =\lambda^{\Delta p_{j+1}} \operatorname{Ch}\left(U D_{j}, \lambda-r\right) \\
& =\lambda^{\Delta p_{j+1}} \prod_{i=0}^{j}(\lambda-r(i+1))^{\Delta p_{j-i}} \quad \text { (by (43)) } \\
& =\prod_{i=0}^{j+1}(\lambda-r i)^{\Delta p_{j+1-i}},
\end{aligned}
$$

and (43) follows for all $j$ by induction. 
4.2. Corollary. For any differential poset $P$, the linear transformation $D$ is surjective, and $U$ is injective.

Proof. It suffices to show that for each $j \in \mathbb{N}, D_{j+1}$ is surjective and $U_{j}$ is injective. Since $D U=U D+r I$, we have by Theorem 4.1 that

$$
\mathrm{Ch}\left(D U_{j}\right)=\prod_{i=0}^{j}(\lambda-r(i+1))^{\Delta p_{j-i}} .
$$

Hence $D U_{j}$ has no zero eigenvalues, so is invertible. Thus $D_{j+1}$ is surjective and $U_{j}$ is injective.

4.3. Corollary. Every differential poset $P$ satisfies $p_{0} \leq p_{1} \leq p_{2} \leq \cdots$.

Proof. Since $U: K P_{j} \rightarrow K P_{j+1}$ is injective we have $p_{j} \leq p_{j+1}$. Or use (43), since the multiplicity of an eigenvalue is nonnegative.

If $0 \leq i \leq j$, then write

$$
P_{[i, j]}=P_{i} \cup P_{i+1} \cup \cdots \cup P_{j}=\{x \in P: i \leq \rho(x) \leq j\} .
$$

Thus $P_{[i, j]}$ is a rank-selected subposet of $P$ in the sense of [St6, p. 131], and the number of maximal chains in $P_{[i, j]}$ is $\alpha(i \rightarrow j)$. We abbreviate $P_{[i]}$ as $P_{i}$ (agreeing with our previous notation) and $P_{[j-1, j]}$ as $P_{j-1, j}$. For the terminology used in the next corollary, see e.g. [St7].

4.4. Corollary. Every differential poset can be partitioned into saturated infinite chains. As a consequence, for any $0 \leq i \leq j$ the poset $P_{[i, j]}$ has the $k$-Sperner property for all $1 \leq k \leq j-i+1$.

Proof. Since $p_{0} \leq p_{1} \leq \cdots$ and $U$ is injective, it follows just as in the proof of [St7, Lemma 1.1] that $P$ can be partitioned into saturated infinite chains. Thus $P_{[i, j]}$ can be partitioned into saturated chains, all going from $P_{s}$ to $P_{j}$ for some $s$. It is then a standard simple argument that $P_{[i, j]}$ has the $k$-Sperner property for $1 \leq k \leq j-i+1$.

4.5. Corollary. Let $P$ be an $r$-differential poset and $Q$ an s-differential poset with $p_{i}=\# P_{i}$ and $q_{i}=\# Q_{i}$, and let $i, j \in \mathbb{P}$. Suppose that $P_{i-1, i} \cong Q_{j-1, j}$ (as posets or simply as graphs, where we identify $P_{i-1, i}$ and $Q_{j-1, j}$ with their Hasse diagrams). Then one of the following two alternatives is true:

(a) $r=s, i=j$, and $p_{k}=q_{k}$ for $0 \leq k \leq j$.

(b) One of $(r, i)$ and $(s, j)$ is equal to $(1,2)$ and the other is equal to $(2,1)$. Moreover, $P_{i-1, i}$ and $Q_{j-1, j}$ are isomorphic to the complete bipartite graph $K_{1,2}$.

Proof. We will show that, except in the situation of (b), we can recover the numbers $r, i, p_{0}, p_{1}, \ldots, p_{i}$ from $P_{i-1, i}$. It is a simple consequence of properties (D1) and (D2) that $P_{i-1, i}$ is connected. Hence if $p_{i}>p_{i-1}$ then (since $p_{i} \geq p_{i-1}$ always) we can determine the decomposition $P_{i-1, i}=P_{i-1} \cup P_{i}$ and 
thus the linear transformation $U D_{i}$. If $p_{i-1}=p_{i}$ then we cannot distinguish between $U D_{i}$ and $D U_{i-1}$, but in this case $\operatorname{Ch}\left(U D_{i}\right)=\operatorname{Ch}\left(D U_{i-1}\right)$. Thus it is always possible to compute $\mathrm{Ch}\left(U D_{i}\right)$ from $P_{i-1, i}$, even if we are only given $P_{i-1, i}$ as an (undirected) graph.

Now note that $\operatorname{Ch}\left(U D_{i}\right)$ has degree at least one, and $\operatorname{deg} \operatorname{Ch}\left(U D_{i}\right)=1$ if and only if $r=1$ and $i=1$ (so $P_{1}$ has one element). Hence we can assume $\operatorname{deg} \operatorname{Ch}\left(U D_{i}\right) \geq 2$.

By Theorem 4.1 the largest eigenvalue $\alpha_{1}$ of $U D_{i}$ is $r i$, and this eigenvalue has multiplicity $p_{0}=1$. Since by assumption $\operatorname{deg} \operatorname{Ch}\left(U D_{i}\right) \geq 2$, there is at least one additional eigenvalue. Again by Theorem 4.1, the second largest eigenvalue $\alpha_{2}$ is $r(i-1)$ with multiplicity $p_{1}-p_{0}$, unless $p_{1}=p_{0}$. Now $p_{1}=p_{0} \quad(=1)$ if and only if $r=1$. It is then evident that $p_{2}=2$, so in this case $\alpha_{2}=r(i-2)$ with multiplicity $p_{2}-p_{1}=1$. It follows that if $\alpha_{2}$ has multiplicity larger than one, then $\alpha_{2}=r(i-1)$. From $\alpha_{1}=r i$ and $\alpha_{2}=r(i-1)$ we can determine $i$ and $r$. Then from (43) we can easily recover $p_{0}, p_{1}, \ldots, p_{i}$.

There remains the case that $\alpha_{2}$ has multiplicity one, i.e., $r=1$ or 2 . Now when $r=2$ it is easily seen that $p_{0}=1, p_{1}=2, p_{2}=5$. Thus either $i=1$ and $P_{i-1, i} \cong K_{1,2}$, or else there is a third largest eigenvalue $\alpha_{3}=r(i-2)$ of multiplicity $p_{2}-p_{1}=3$. Similarly when $r=1$ we see that $p_{0}=1, p_{1}=1$, $p_{2}=2, p_{3}=3$. Thus either $i=1$ (which is equivalent to $\operatorname{deg} \operatorname{Ch}\left(U D_{i}\right)=1$ ), or $i=2$ (in which case $\left.P_{i-1, i} \cong K_{1,2}\right)$, or there is a third largest eigenvalue $\alpha_{3}=r(i-3)$ of multiplicity $p_{3}-p_{2}=1$. Hence unless $(r, i)=(1,2)$ or $(2,1)$ (in which case $\left.P_{i-1, i} \cong K_{1,2}\right)$ or $(r, i)=(1,1)$ (so $\operatorname{deg} \operatorname{Ch}\left(U D_{i}\right)=1$ ), we can determine whether $\alpha_{3}=r(i-2)$ or $\alpha_{3}=r(i-3)$. From this and $\alpha_{1}=r i$ we can determine $r$ and $i$, and then get $p_{0}, \ldots, p_{i}$ from (43) as before.

We can compute the eigenvectors of $U D_{j}$ in an inductive way, as described by the next result.

4.6. Proposition. Let $E_{j}(r i)$ denote the eigenspace of $U D_{j}$ belonging to the eigenvalue $r i$. Then

$$
\begin{aligned}
E_{j}(0) & =\operatorname{ker} D_{j}:=\left\{f \in K P_{j}: D f=0\right\} \\
& =\left(U P_{j-1}\right)^{\perp}:=\left\{f \in K P_{j}:\langle f, U g\rangle=0 \text { for all } g \in K P_{j-1}\right\}
\end{aligned}
$$

Moreover, $E_{j}(r i)=U^{i} E_{j-i}(0)$ for $1 \leq i \leq j$. In particular, the eigenvector belonging to the largest eigenvalue $j r$ (or the unique positive eigenvector, up to scalar multiplication) is given by $\sum_{x \in P_{j}} e(x) x$, where $e(x)$ is as in (15).

Proof. Since $U$ is injective, $\operatorname{ker} U D_{j}=\operatorname{ker} D_{j}$. Now for $f \in K P_{j}$,

$$
\begin{aligned}
\langle f, U g\rangle & =0 \quad \text { for } g \in K P_{j-1} \\
& \Leftrightarrow\langle D f, g\rangle=0 \text { for all } g \in K P_{j-1} \\
& \Leftrightarrow D f=0 .
\end{aligned}
$$

This proves (44). 
Let $f \in E_{j-i}(0)$. Then

$$
\begin{aligned}
U D\left(U^{i} f\right) & =r i U^{i} f+U^{i+1} D f \quad(\text { by Corollary 2.4(a)) } \\
& =r i U^{i} f .
\end{aligned}
$$

Hence $U^{i} E_{j-i}(0) \subseteq E_{j}(r i)$. But (since $U$ is injective) $\operatorname{dim} U^{i} E_{j-i}(0)=$ $\operatorname{dim} E_{j-i}(0)=\Delta p_{j-i}$, while by (43) $\operatorname{dim} E_{j}(r i) \leq \Delta p_{j-i}$. (Actually, it follows from (43) that $\operatorname{dim} E_{j}(r i)=\Delta p_{j-i}$ since $U D_{j}$ is selfadjoint.) Hence $E_{j}(r i)=U^{i} E_{j-i}(0)$, as desired.

For Young's lattice we can be even more explicit about the eigenvectors of $U D_{j}$. We use notation and terminology from [M] throughout.

4.7. Proposition. Let $\chi^{\lambda}$ denote the irreducible character of the symmetric group $S_{j}$ corresponding to the partition $\lambda$ of $j$. Then for any partition $\mu$ of $j$, the vector

$$
X_{\mu}=\sum_{\lambda \vdash j} \chi^{\lambda}(\mu) \lambda
$$

is an eigenvector for $U D_{j}: K Y_{j} \rightarrow K Y_{j}$ belonging to the eigenvalue $m_{1}(\mu)$ (the number of parts of $\mu$ equal to 1$)$. Moreover, the $X_{\mu}$ 's give a complete set of orthogonal eigenvectors for $U D_{j}$.

Proof. We use the "representation" of Young's lattice discussed after the proof of Theorem 2.5. An eigenvector of $U D_{j}$ belonging to the eigenvalue $i$ then corresponds to a symmetric function $f$ of degree $j$ satisfying

$$
p_{1} \frac{\partial}{\partial p_{1}} f=i f \text {. }
$$

But the power sum symmetric function $p_{\mu}=p_{1}^{m_{1}(\mu)} p_{2}^{m_{2}(\mu)} \ldots$ clearly satisfies

$$
p_{1} \frac{\partial}{\partial p_{1}} p_{\mu}=m_{1}(\mu) p_{\mu}
$$

Now by $[\mathrm{M},(7.8)]$,

$$
p_{\mu}=\sum_{\lambda} \chi^{\lambda}(\mu) s_{\lambda}
$$

Since $\lambda \in Y_{j}$ corresponds to the Schur function $s_{\lambda}$, it follows that $U D_{j} X_{\mu}=$ $m_{1}(\mu) X_{\mu}$ as desired. Completeness and orthogonality follow from the fact that the $p_{\mu}$ 's with $\mu \vdash k$ form a basis for all symmetric functions of degree $k$ (over a field of characteristic 0$)[\mathrm{M},(2.12)]$, and that the $p_{\mu}$ 's are orthogonal with respect to the scalar product defined by $\left\langle s_{\lambda}, s_{\mu}\right\rangle=\delta_{\lambda \mu}[\mathrm{M},(4.7)]$.

Note. One could also give a more straightforward but less elegant proof of Proposition 4.7 by using known properties of the characters of $S_{j}$ to show directly that the $X_{\mu}$ 's satisfy the conditions necessary to deduce from Proposition 4.6 that they are eigenvectors of $U D_{j}$. 
Since $U D_{j}: K Y_{j} \rightarrow K Y_{j}$ has multiple eigenvalues, the eigenvectors $X_{\mu}$ of (45) are not unique. In other words, we cannot determine the character values $\chi^{\lambda}(\mu)$ directly from $U D_{j}$. This suggests the problem of finding additional selfadjoint operators commuting with $U D_{j}$ for which the $X_{\mu}$ 's are unique simultaneous eigenvectors. Once again the theory of symmetric functions provides a solution. Given $i \in \mathbb{P}$, define continuous linear transformations $U(i): \widehat{K} Y \rightarrow \widehat{K} Y$ as follows: If $\lambda \in Y$, then

$$
U(i) \lambda=\sum_{\mu}(-1)^{\mathrm{h}(\mu-\lambda)} \mu, \quad D(i) \lambda=\sum_{\nu}(-1)^{\mathrm{ht}(\lambda-\nu)} \nu,
$$

where $\mu$ ranges over all partitions such that $\lambda \subset \mu$ and $\lambda-\mu$ is a border strip of length $i$ [M, pp. 31-32], and $\nu$ ranges over all partitions such that $\nu \subset \lambda$ and $\lambda-\nu$ is a border strip of length $i$. Moreover, the height $\operatorname{ht}(\theta)$ of a border strip $\theta$ is, following [M], one less than the number of rows it occupies. Clearly $U(i)$ and $D(i)$ are adjoint with respect to the pairing $\langle$,$\rangle .$

4.8. Proposition. The transformations $U(i) D(i), i \geq 1$, pairwise commute. The vector $X_{\mu}$ of $(45)$ is an eigenvector for $U(i) D(i)_{j}$ with eigenvalue $i_{i}(\mu)$, where $m_{i}(\mu)$ is the number of parts of $\mu$ equal to $i$. Hence (since $\mu$ is determined by the $m_{i}(\mu)$ 's) the vectors $X_{\mu}$ for $\mu \vdash j$ are the unique simultaneous eigenvectors for $U(i) D(i)_{j}, \quad 1 \leq i \leq j$.

Proof. We represent elements of $K Y_{j}$ by symmetric functions of degree $j$, as in the proof of Proposition 4.7. It follows from [M, Example 11, pp. 31-32] that $U(i)$ corresponds to multiplication by $p_{i}$, and from [M, Example 3(c), p. 44] that $D(i)$ corresponds to the operator $i \partial / \partial p_{i}$. Hence the $U(i) D(i)$ 's commute. Moreover, from the proof of Proposition 4.7 we have that $X_{\mu}$ corresponds to $p_{\mu}$. Since

$$
p_{i} i \frac{\partial}{\partial p_{i}} p_{\mu}=i m_{i}(\mu) p_{\mu}
$$

the proof follows.

Equation (43) suggests the problem of computing the characteristic polynomials of other linear transformations $K P_{j} \rightarrow K P_{j}$ defined by power series in $U$ and $D$. Define a continuous endomorphism $\sigma \in \operatorname{End}(\widehat{K} P)$ to be balanced if for all $j \geq 0$ the image $\sigma\left(K P_{j}\right)$ of $K P_{j}$ is contained in $K P_{j}$. Clearly a word $w \in\{U, D\}^{*}$ is balanced if and only if it contains the same number of $U$ 's and $D$ 's. Moreover, if a power series $f(U, D)=\sum c_{w} w$ defines a balanced endomorphism of $\widehat{K} P$, then we do not change the endomorphism by deleting all terms $c_{w} w$ in $f(U, D)$ for which $w$ is not balanced. (For instance, $D U^{2}-U D U-r U$ is balanced, so $D U^{2}-U D U-r U=0$.) Hence we can assume a balanced endomorphism $f(U, D)$ is an (infinite) linear combination of balanced words. 
4.9. Proposition. Let $s(n, k)$ and $S(n, k)$ denote Stirling numbers of the first and second kinds, respectively. Then

$$
\begin{aligned}
& (U D)^{n}=\sum_{k=0}^{n} r^{n-k} S(n, k) U^{k} D^{k}, \\
U^{n} D^{n}= & \sum_{k=0}^{n} r^{n-k} s(n, k)(U D)^{k} \\
= & U D(U D-r)(U D-2 r) \cdots(U D-(n-1) r) .
\end{aligned}
$$

Proof. Equation (46) is an immediate consequence of [C, Exercise 2, p. 220]. It is also easy to prove by induction on $n$, using Corollary 2.4 (a) and the recurrence [C, Theorem A, p. 208; St6, (23), p. 33]

$$
S(n+1, k)=k S(n, k)+S(n, k-1) .
$$

(47) and (48) are then immediate consequences of standard properties of Stirling numbers [C, equation 5c, p. 213; St6, p. 35].

4.10. Lemma. Let $f(U, D)$ be a power series over $K$ which defines a balanced endomorphism of $\widehat{K} P$. Then $f(U, D)$ can be expressed as a power series in $U D$ over $K$, i.e.,

$$
f(U, D)=\sum_{n \geq 0} a_{n}(U D)^{n}, \quad a_{n} \in K .
$$

Proof. When we use Corollary $2.4($ a) to write $f(U, D)$ in the form (37), we do not alter the property of being balanced. Hence we can write

$$
f(U, D)=\sum_{n \geq 0} b_{n} U^{n} D^{n}, \quad b_{n} \in K .
$$

Using (47) we can then write $f(U, D)$ in the form (49).

4.11. Corollary. Let $P$ be a differential poset. Then

(a) The algebra of all power series $f(U, D)$ over $K$ which define balanced endomorphisms of $\widehat{K} P$ is commutative.

(b) If $f(U, D)$ is as in (a), then $f$ is selfadjoint with respect to the pairing $\langle$,$\rangle , i.e., \langle f(U, D) u, v\rangle=\langle u, f(U, D) v\rangle$ for all $u \in \widehat{K} P, v \in K P$.

Proof. (a) Immediate from (49).

(b) The word $U D$, and hence $(U D)^{n}$, is clearly selfadjoint, so by the linearity and continuity of taking adjoints the series (49) is also selfadjoint.

Corollary 4.11(b) has the following combinatorial significance. Let $l_{1}, l_{2}$, $\ldots, l_{2 n}$ be a sequence of the symbols $<$ and $>$ with $n<$ 's and $n>$ 's. Let $x, y \in P_{n}$ for some $n$ (where $P$ is of course a differential poset). Then the number of Hasse walks

$$
x=x_{0} l_{1} x_{1} l_{2} x_{2} \cdots l_{2 n} x_{2 n}=y
$$


is equal to the number

$$
y=y_{0} l_{1} y_{1} l_{2} y_{2} \cdots l_{2 n} y_{2 n}=x .
$$

4.12. Proposition. Let $P$ be an $r$-differential poset with $p_{i}=\# P_{i}$, and let $f(U, D)$ be a power series over $K$ which defines a balanced endomorphism of $\widehat{K} P$. Write

$$
f(U, D)=\sum_{n} b_{n}(U D)^{n}
$$

and define

$$
\alpha_{i}=\sum_{n} b_{n}(r i)^{n}
$$

or equivalently,

$$
\sum_{i \geq 0} \frac{\alpha_{i} t^{i}}{i !}=\left.f\left(r z, \frac{d}{d z}\right) e^{t z}\right|_{z=1} .
$$

Then the characteristic polynomial of the linear transformation $f(U, D): K P_{j}$ $\rightarrow K P_{j}$ is given by

$$
\operatorname{Ch} f(U, D)_{j}=\prod_{i=0}^{j}\left(\lambda-\alpha_{i}\right)^{\Delta p_{j-i}} .
$$

Moreover, the space $E_{j}(r i)$ of Proposition 4.6 is an eigenspace for the eigenvalue $\alpha_{i}$.

Proof. Let $A: V \rightarrow V$ be any linear transformation on a finite-dimensional $K$ vector space $V$, and let $f(A)$ be a formal power series over $K$ which defines a linear transformation $V \rightarrow V$. If $\operatorname{Ch} A=\prod\left(\lambda-\alpha_{i}\right)$, then $\operatorname{Ch} f(A)=$ $\Pi\left(\lambda-f\left(\alpha_{i}\right)\right)$. Hence (53) follows from (50), (51), and (43). Moreover, if a vector $v \in V$ satisfies $A v=\alpha v$, then $f(A) v=f(\alpha) v$. Hence the statement about $E_{j}(r i)$ follows.

To show (52) it suffices by linearity and continuity to assume $f(U, D)=$ $(U D)^{n}$, so $\alpha_{i}=(r i)^{n}$. But it is routine to see that

$$
\left(r z \frac{d}{d z}\right)^{n} e^{t z}=\sum_{i \geq 0} \frac{(r i)^{n} z^{i} t^{i}}{i !}
$$

and the proof follows after setting $z=1$.

4.13. Example. Suppose $f(U, D)=U^{n} D^{n}$. Then by the previous proposition and (48), we get

$$
\mathrm{Ch}\left(U^{n} D^{n}\right)_{j}=\prod_{i=0}^{j}\left(\lambda-r^{n} i(i-1) \cdots(i-n+1)\right)^{\Delta p_{j-i}} .
$$

Note that the eigenvalue 0 has multiplicity $p_{j}-p_{j-n}$, so $\operatorname{rank}\left(U^{n} D^{n}\right)_{j}=$ $p_{j-n}$ since $U^{n} D^{n}$ is selfadjoint. This is consistent with the observation that 
$\operatorname{rank}\left(U^{n} D^{n}\right)_{j}=\operatorname{rank}\left(D^{n}\right)_{j}$ since $U^{n}$ is injective, and $\operatorname{rank}\left(D^{n}\right)_{j}=p_{n-j}$ since $D^{n}$ is surjective.

Our last topic of this section will be the computation of the spectrum (eigenvalues of the adjacency matrix) of certain graphs associated with differential posets. For any locally finite poset $Q$, define the Hasse graph $\mathscr{H}(Q)$ to be the (undirected) graph with vertex set $Q$, and with $x, y \in Q$ joined by an edge if $x$ covers $y$ or $y$ covers $x$. Let $A(G)$ denote the adjacency matrix of $G$ [B-C-L; C-D-S] and write

$$
\operatorname{Ch} G=\operatorname{Ch} A(G)=\operatorname{det}(\lambda I-A(G)) .
$$

Regarding $r$ as fixed, define for $1 \leq a \leq b-1$ the $(b-a+2) \times(b-a+2)$ tridiagonal matrix

$$
M_{a b}=\left[\begin{array}{cccccccc}
0 & a r & 0 & & 0 & & \\
1 & 0 & (a+1) r & & 0 & & \\
0 & 1 & 0 & & \ddots & & & \\
& & & \ddots & & & & \\
& & \ddots & & & 0 & b r \\
& & & & & & 1 & 0
\end{array}\right] \text {, }
$$

and write $C_{a b}=\operatorname{Ch} M_{a b}$.

4.14. Theorem. Let $P$ be an $r$-differential poset with $p_{i}=\# P_{i}$, and let $0 \leq$ $i \leq j$. Then

$$
\operatorname{Ch} \mathscr{H}\left(P_{[i, j]}\right)=\left(\prod_{b=0}^{j-i} C_{1 b}^{\Delta p_{j-h}}\right) \cdot\left(\prod_{b=j-i+1}^{j} C_{b-j+i+1, b}^{\Delta p_{j-h}}\right) .
$$

Proof. Regard $i$ and $j$ as fixed throughout. Set

$$
s^{*}=\left\{\begin{array}{l}
1, \quad i<s \leq j \\
i-s+1, \quad 0 \leq s \leq i .
\end{array}\right.
$$

Let $A$ denote the adjacency matrix of $\mathscr{H}\left(P_{[i, j]}\right)$. Let $v \in K P_{[i, j]}$, and write (uniquely)

$$
v=v_{i}+v_{i+1}+\cdots+v_{j}, \quad v_{s} \in K P_{s} .
$$

Then $A$ acts on the vector space $K P_{[i, j]}$ by

$$
\begin{aligned}
A v= & D v_{i+1}+\left(U v_{i}+D v_{i+2}\right)+\left(U v_{i+1}+D v_{i+3}\right) \\
& +\cdots+\left(U v_{j-2}+D v_{j}\right)+U v_{j-1} .
\end{aligned}
$$

Let $0 \neq v \in K P_{s}$ and $D v=0$, where $i<s \leq j$. Let $T=\left[\tau_{1}, \tau_{2}, \ldots, \tau_{j-s+1}\right]^{t}$ be an eigenvector of $M_{1, j-s}=M_{s^{*}, j-s}$ belonging to the eigenvalue $\alpha$. We claim that the element

$$
E(v, T)=\tau_{1} v+\tau_{2} U v+\cdots+\tau_{j-s+1} U^{j-s} v
$$


is an eigenvector of $A$ belonging to $\alpha$. For using (55), we have

$$
\begin{aligned}
A E(v, T)= & (U+D)\left(\tau_{1} v+\tau_{2} U v+\cdots+\tau_{j-s} U^{j-s-1} v\right)+\tau_{j-s+1} D U^{j-s} v \\
= & \tau_{1} U v+\tau_{2}\left(U^{2} v+r v\right)+\tau_{3}\left(U^{3} v+2 r U v\right) \\
& +\cdots+\tau_{j-s}\left(U^{j-s} v+(j-s-1) r U^{j-s-2} v\right) \\
& +\tau_{j-s+1}(j-s) r U^{j-s-1} v
\end{aligned}
$$

using Corollary 2.4(a) and $D v=0$. Rearranging yields

$$
\begin{aligned}
A E(v, T)= & \tau_{2} r v+\left(\tau_{1}+2 r \tau_{3}\right) U v+\left(\tau_{2}+3 r \tau_{4}\right) U^{2} v \\
& +\cdots+\left(\tau_{j-s-1}+(j-s) r \tau_{j-s+1}\right) U^{j-s-1} v \\
& +\tau_{j-s} U^{j-s} v .
\end{aligned}
$$

Now $M_{1, j-s} T=\alpha T$ means that

$$
r \tau_{2}=\alpha \tau_{1}, \quad \tau_{1}+2 r \tau_{3}=\alpha \tau_{2}, \ldots, \tau_{j-s}=\alpha \tau_{j-s+1} .
$$

Thus (57) becomes $A E(v, T)=\alpha E(v, T)$, proving the claim.

Similarly let $v \in K P_{s}$ and $D v=0$, where $0 \leq s \leq i$, and let $T=$ $\left[\tau_{1}, \tau_{2}, \ldots, \tau_{j-i+1}\right]^{t}$ be an eigenvector of $M_{i-s+1, j-s}=M_{s^{*}, j-s}$ belonging to $\alpha$. Then analogous reasoning (which we omit) shows that the element

$$
E(v, T)=\tau_{1} U^{i-s} v+\tau_{2} U^{i-s+1} v+\cdots+\tau_{j-i+1} U^{j-s} v
$$

is an eigenvector of $A$ belonging to $\alpha$.

We now claim the following two facts.

(a) The matrices $M_{a b}$ are diagonalizable so they have $b-a+2$ linearly independent eigenvectors. (In fact, $M_{a b}$ has distinct eigenvalues.)

(b) Denote by $\mathscr{E}_{s}$ a complete set of linearly independent eigenvectors of $M_{s^{*}, j-s}$, for $0 \leq s \leq j$. Given $T \in \mathscr{E}_{s}$, let $E(v, T)$ denote the corresponding eigenvector (56) or (58) of $A$ (where $0 \neq v \in K P_{s}$ and $D v=0$ ). Let $\mathscr{B}_{s}$ be a basis for $\left\{v \in K P_{s}: D v=0\right\}$. Then all the eigenvectors $E(v, T)$, where $0 \leq s \leq j, v \in \mathscr{B}_{s}$, and $T \in \mathscr{E}_{s}$, form a complete set of $p_{i}+p_{i+1}+\cdots+p_{j}$ linearly independent eigenvectors of $A$.

To prove (a), G. Strang has pointed out to me that the diagonal matrix

$$
D=\operatorname{diag}\left[1,(a r)^{1 / 2},\left(a(a+1) r^{2}\right)^{1 / 2}, \ldots,\left(a(a+1) \cdots b r^{b-a-1}\right)^{1 / 2}\right]
$$

has the property that $D M_{a b} D^{-1}$ is symmetric. Hence $M_{a b}$ is diagonalizable. (It follows from the theory of tridiagonal matrices that in fact $M_{a b}$ has distinct eigenvalues; see [M-M, Chapter III.3.7.1].)

To show (b), let $V_{s}=\left\{v \in K P_{s}: D v=0\right\}$. Since $D$ is surjective, we have $\operatorname{dim} V_{s}=\Delta p_{s}$. We claim that

$$
K P_{s}=V_{s} \oplus U V_{s-1} \oplus \cdots \oplus U^{s} V_{0} \quad \text { (vector space direct sum) }
$$


(59) is trivial for $s=0$. Assuming for $s-1$, by (44) we have

$$
\begin{aligned}
K P_{s} & =V_{s} \oplus U\left(K P_{s-1}\right) \\
& =V_{s} \oplus U\left(V_{s-1} \oplus U V_{s-2}+\cdots+U^{s-1} V_{0}\right) \\
& =V_{s} \oplus U V_{s-1} \oplus \cdots \oplus U^{s} V_{0},
\end{aligned}
$$

since $U$ is injective. Thus (59) is proved.

Now a straightforward argument shows that if $T=\left[\tau_{1}, \ldots, \tau_{b-a+1}, 0\right]^{t}$ and $M_{a b} T=\alpha T$, then $T=0$. For $0 \leq s \leq j$ and $v \in \mathscr{B}_{s}$ as above, let $W_{j}(v, s)$ denote the span of the projections onto $K P_{j}$ (with respect to the scalar product $\langle$,$\left.\rangle on K P_{[i, j]}\right)$ of the vectors $E(v, T)$, where $T \in \mathscr{E}_{s}$. Thus by (56), (58), and the first sentence of this paragraph, we have that $W_{j}(v, s)$ is spanned by vectors $U^{j-s} v$ where $v \in V_{s}$. By (59) the subspaces $W_{j}(v, s)$ are linearly independent. Hence if $W(v, s)$ denotes the span of the vectors $E(v, T)$ themselves, then the subspaces $W(v, s)$ are linearly independent. Thus to complete the proof of (b), we need to show that for fixed $0 \leq s \leq j$ and $v \in V_{s}$, the vectors $E(v, T)$ are linearly independent (where $T$ ranges over $\mathscr{E}_{s}$ ), and that we have found a total of $\# P_{[i, j]}$ linearly independent eigenvectors.

Since $v \neq 0, U$ is injective, and $U$ maps $K P_{a}$ to $K P_{a+1}$, we have that the vectors $U^{s^{*}} v, U^{s^{*}-1} v, \ldots, U^{j-s} v$ (for $0 \leq s \leq j$ ) are linearly independent. Since the vectors $T \in \mathscr{E}_{s}$ are linearly independent by definition, it follows from the definition (56) and (58) of $E(v, T)$ that the vectors $E(v, T)$, where $T \in \mathscr{E}_{s}$, are linearly independent. Hence the vectors $E(v, T)$ for $0 \leq s \leq j$, $v \in \mathscr{B}_{s}, T \in \mathscr{E}_{s}$, are linearly independent eigenvectors for $A$.

Now $\# \mathscr{B}_{s}=\Delta p_{s}\left(\right.$ since $D: K P_{s} \rightarrow K P_{s-1}$ is surjective $)$, and

$$
\# \mathscr{E}_{s}= \begin{cases}j-i+1, & 0 \leq s \leq i, \\ j-s+1, & i<s \leq j .\end{cases}
$$

Thus we have found a total of

$$
\sum_{s=0}^{j}\left(\# \mathscr{B}_{s}\right)\left(\# \mathscr{E}_{s}\right)=p_{i}+p_{i+1}+\cdots+p_{j}
$$

linearly independent eigenvectors of $A$, completing the proof of (b).

If $T$ belongs to the eigenvalue $\alpha$ of $M_{s^{*}, j-s}$ (for $0 \leq s \leq j$ ), then $E(v, T$ ) also belongs to the eigenvalue $\alpha$ of $A$. Thus the eigenvector $T$ leads to a total of $\operatorname{dim} V_{s}=\Delta p_{s}$ occurrences of the eigenvalue $\alpha$ of $A$. (We cannot say that $\alpha$ has multiplicity $\Delta p_{s}$, since it can happen that another eigenvalue $\alpha^{\prime}$ of some different $M_{a b}$ could equal $\alpha$.) Hence each $s$ contributes a factor $\left(\mathrm{Ch} M_{s^{*}, j-s}\right)^{\Delta p_{s}}$ to $\mathrm{Ch} \mathscr{H}\left(P_{[i, j]}\right)$, and (54) follows.

For small values of $j-i$ we have the following more explicit formulas for $\mathrm{Ch} \mathscr{H}\left(P_{[i, j]}\right)$, obtained by computing each $C_{s^{*}, j-s}$.

$$
\operatorname{Ch} \mathscr{H}\left(P_{[j, j]}\right)=\lambda^{p_{j}},
$$




$$
\begin{gathered}
\operatorname{Ch} \mathscr{H}\left(P_{[j-1, j]}\right)=\lambda^{\Delta p_{j}} \prod_{s=1}^{j}\left(\lambda^{2}-r s\right)^{\Delta p_{j-s}}, \\
\operatorname{Ch} \mathscr{H}\left(P_{[j-2, j]}\right)=\lambda^{\Delta p_{j}}\left(\lambda^{2}-r\right)^{\Delta p_{j-1}} \prod_{s=2}^{j}\left(\lambda^{3}-r(2 s-1) \lambda\right)^{\Delta p_{j-s}}, \\
\operatorname{Ch} \mathscr{H}\left(P_{[j-3, j]}\right)=\lambda^{\Delta p_{j}}\left(\lambda^{2}-r\right)^{\Delta p_{j-1}}\left(\lambda^{3}-3 r \lambda\right)^{\Delta p_{j-2}} \\
\cdot \prod_{s=3}^{j}\left(\lambda^{4}-3 r(s-1) \lambda^{2}+r^{2} s(s-2)\right)^{\Delta p_{j-s}}, \\
\operatorname{Ch} \mathscr{H}\left(P_{[j-4, j]}\right)=\lambda^{\Delta p_{j}}\left(\lambda^{2}-r\right)^{\Delta p_{j-1}}\left(\lambda^{3}-3 r \lambda\right)^{\Delta p_{j-2}} \\
\cdot\left(\lambda^{4}-6 r \lambda^{2}+3 r^{2}\right)^{\Delta p_{j-3}} \\
\cdot \prod_{s=4}^{j}\left(\lambda^{5}-2 r(2 s-3) \lambda^{3}+3 r^{2}\left(s^{2}-3 s+1\right) \lambda\right)^{\Delta p_{j-s}} .
\end{gathered}
$$

It is easy to see that

$$
\operatorname{rank} M_{a b}= \begin{cases}b-a+2, & \text { if } a \equiv b(\bmod 2), \\ b-a+1, & \text { if } a \neq \equiv b(\bmod 2) .\end{cases}
$$

It then follows from Theorem 4.14 that the adjacency matrix $A$ of $\mathscr{H}\left(P_{[i, j]}\right)$ satisfies

$$
\operatorname{rank} A= \begin{cases}2\left(p_{j-1}+p_{j-3}+\cdots+p_{i+1}\right), & \text { if } i \equiv j(\bmod 2), \\ 2\left(p_{j-1}+p_{j-3}+\cdots+p_{i}\right), & \text { if } i \not \equiv j(\bmod 2)\end{cases}
$$

Note that the proof of Theorem 4.14 determines not only the eigenvalues of $A$, but also the eigenvectors (assuming we know the eigenvectors of $M_{a b}$ and a basis $\mathscr{B}_{s}$ for $\left.V_{s}=\left\{v \in K P_{s}: K v=0\right\}\right)$. In particular, let $T=$ $\left[\tau_{1}, \tau_{2}, \ldots, \tau_{j-i+1}\right]^{t}$ be the unique (up to scalar multiplication) nonnegative eigenvector of $M_{i+1, j}$ (i.e., the eigenvector belonging to the largest eigenvalue of $\left.M_{i+1, j}\right)$. Then the unique nonnegative eigenvector of $A$ is given by

$$
\sum_{s=i}^{j} \tau_{s-i+1} \sum_{x \in P_{s}} e(x) x .
$$

Remark. For this remark we assume familiarity with the Brauer algebra $\mathscr{A}_{f}=$ $\mathscr{A}_{f}^{(x)}$ as defined in [H-W]. $\mathscr{A}_{f}$ is a complex semisimple algebra with a filtration of two-sided ideals

$$
\mathscr{A}_{f}=\mathscr{A}_{f}(0) \supset \mathscr{A}_{f}(2) \supset \cdots \supset \mathscr{A}_{f}\left(2\left[\frac{f+2}{2}\right]\right)=(0) .
$$

Let $\mathscr{Q}_{f}(s)=\mathscr{A}_{f} / \mathscr{A}_{f}(2 s)$. In particular, $\mathscr{Q}_{f}(1) \cong \mathbb{C} S_{f}$, the group algebra of the symmetric group $S_{f}$. There is also a natural inclusion $\mathscr{Q}_{f-1}(s) \subset \mathscr{Q}_{f}(s)$. Let $Y$ denote Young's lattice. What we wish to note here is that the graph 
$\mathscr{H}\left(Y_{[j-2 s+1, j]}\right)$ is just the "Brattelli diagram" of the pair $\mathscr{Q}_{j-1}(s) \subset \mathscr{Q}_{j}(s)$. (If $j$ is even and $s=(j+2) / 2$, then we set $\left.Y_{[-1, j]}=Y_{[0, j]}.\right)$ See, for instance, [G-H-J], where the significance of the eigenvalues and eigenvectors of Brattelli diagrams is briefly discussed.

\section{FibonACCI DIFFERENTIAL POSETS}

An obvious problem at this point is to produce further examples of differential posets. Let us first note the following result, whose proof is routine and will be omitted.

5.1. Proposition. Suppose $P$ is an $r$-differential poset and $Q$ is an s-differential poset. Then $P \times Q$ is an $(r+s)$-differential poset.

We will call a differential poset $P$ irreducible if it cannot be written as a product $P_{1} \times P_{2}$ of differential posets $P_{i}$. To classify the differential posets it of course suffices to find all irreducible ones. The purpose of this section is to describe and analyze a certain irreducible $r$-differential poset, denoted $Z(r)$, which has some interesting properties.

Let $A_{r}$ be an alphabet of $r+1$ letters. We will regard the letters as consisting of the number 1 with $r$ different "colors," denoted $1_{1}, 1_{2}, \ldots, 1_{r}$, together with the number 2 . Thus $A_{r}=\left\{1_{1}, 1_{2}, \ldots, 1_{r}, 2\right\}$. Let $A_{r}^{*}$ denote the free monoid generated by $A_{r}$, i.e., $A_{r}^{*}$ consists of all finite words $a_{1} a_{2} \cdots a_{k}$ (including the empty word $\varnothing$ ) of elements of $A_{r}$.

5.2. Definition. Let $r \in \mathbb{P}$. Define a poset $Z(r)$ as follows. As a set, $Z(r)$ coincides with $A_{r}^{*}$. If $w \in Z(r)$, then define $w^{\prime}$ to be covered by $w$ in $Z(r)$ if either:

(a) $w^{\prime}$ is obtained from $w$ by changing a 2 to some $1_{i}$, provided that the only letters to the left of this 2 are also 2 's, or

(b) $w^{\prime}$ is obtained from $w$ by deleting the first letter of the form $1_{i}$.

This defines the cover relations in $Z(r)$, and hence by transitivity a partial ordering of $Z(r)$. We call $Z(r)$ the Fibonacci $r$-differential poset.

Thus if $w=2^{j}$, then $w$ covers the $r j$ elements

$$
C^{-}(w)=\left\{2^{i} 1_{m} 2^{j-1-i}: 0 \leq i \leq j-1,1 \leq m \leq r\right\}
$$

and is covered by the $r(j+1)$ elements

$$
C^{+}(w)=\left\{2^{i} 1_{m} 2^{j-i}: 0 \leq i \leq j, 1 \leq m \leq r\right\}
$$

while if $w=2^{j} 1_{k} v$ for some $v \in Z(r)$, then $w$ covers the $r j+1$ elements

$$
C^{-}(w)=\left\{2^{i} 1_{m} 2^{j-1-i} 1_{k} v: 0 \leq i \leq j-1,1 \leq m \leq r\right\} \cup\left\{2^{j} v\right\},
$$

and is covered by the $r(j+1)+1$ elements

$$
C^{+}(w)=\left\{2^{i} 1_{m} 2^{j-i} 1_{k} v: 0 \leq i \leq j, 1 \leq m \leq r\right\} \cup\left\{2^{j+1} v\right\} .
$$


5.3. Theorem. $Z(r)$ is an irreducible $r$-differential poset, with rank-generating function

$$
F(Z(r), q)=\left(1-r q-q^{2}\right)^{-1} .
$$

Proof. It is easy to see that (D1) is satisfied, with the rank $\rho(w)$ of $w \in Z(r)$ given by the sum of its letters (ignoring the subscripts on the 1 's). From this it is easy to deduce (64). The above expressions (60)-(63) for $C^{-}(w)$ and $C^{+}(w)$ show that (D3) is satisfied; it remains to show (D2) and irreducibility.

If $u$ and $u^{\prime}$ are two unequal elements which cover a common element $w$, then by $(61)$ and (63) we must have one of the following two situations (where $y$ and $v$ denote arbitrary elements of $Z(r))$ :

(a) $w=2^{j} y, u=2^{a} 1_{m} 2^{j-a} y, u^{\prime}=2^{b} 1_{r} 2^{j-b} y \quad(a<b)$,

(b) $w=2^{j} 1_{k} v, u=2^{a} 1_{m} 2^{j-a} 1_{k} v, u^{\prime}=2^{j+1} v$.

In both cases it is easily seen that $u$ and $u^{\prime}$ determine $w$. Thus for any $u$ and $u^{\prime}$, we have $\left|C^{-}(u) \cap C^{-}\left(u^{\prime}\right)\right| \leq 1$. Now in case (a) there is a unique element $w^{\prime}=2^{j+1} y$ which covers both $u$ and $u^{\prime}$; while in case (b) there is a unique element $w^{\prime}=2^{j+1} 1_{k} v$ covering both $u$ and $u^{\prime}$. Hence

$$
\left|C^{-}(u) \cap C^{-}\left(u^{\prime}\right)\right|=1 \Rightarrow\left|C^{+}(u) \cap C^{+}\left(u^{\prime}\right)\right|=1 .
$$

By similar reasoning the converse to (65) is shown. Thus we have proved (D2).

Now note that the element 2 of $Z(r)$ covers exactly $r$ elements, while all other elements $1_{i} 1_{j}$ of $Z(r)$ of rank 2 cover exactly one element. Suppose $Z(r)=P \times Q$, where $|P|>1$ and $|Q|>1$. Then $P$ and $Q$ both have a $\widehat{O}$, and if $P$ has $c$ elements $x_{1}, \ldots, x_{c}$ covering $\hat{O}(1 \leq c \leq r-1)$ then $Q$ has $r-c$ elements $y_{1}, \ldots, y_{r-c}$ covering $\hat{O}$. Thus $r \geq 2$. But then each element $\left(x_{i}, y_{i}\right)$ of $P \times Q$ has rank 2 and covers the two elements $\left(\widehat{O}, y_{j}\right)$ and $\left(x_{i}, \widehat{O}\right)$. This gives a contradiction unless $r=2$ and $c=1$, so assume $r=2$. If $P$ and $Q$ are differential with $P \times Q=Z(2)$, then $P$ and $Q$ must be 1-differential. Any 1-differential poset has a $\hat{O}$, a unique element $x_{1}$ covering $\widehat{O}$, and two elements $x_{2}$ and $x_{2}^{\prime}$ covering $x_{1}$ (by (D3)). By (D2), there is a unique element $y_{3}$ covering both $x_{2}$ and $x_{2}^{\prime}$, and then by (D2) elements $x_{3}$ covering $x_{2}$, and $x_{3}^{\prime}$ covering $x_{2}^{\prime}$. Hence $F(P, q)=1+q+2 q^{2}+3 q^{3}+\cdots$ and $F(Q, q)=1+q+2 q^{2}+3 q^{3}+\cdots$. Thus $F(P \times Q)=F(P, q) F(Q, q)=$ $1+2 q+5 q^{2}+10 q^{3}+\cdots$. But $Z(2)$ has 12 elements of rank 3 , a contradiction. This completes the proof.

Note. The above proof shows that if $r \neq 2$ then $Z(r) \neq P \times Q$ for any nontrivial $P, Q$. However, when $r=2$ we have shown only that $Z(2) \neq P \times Q$ for differential posets $P$ and $Q$. With a little more work we could in fact show $Z(2) \neq P \times Q$ for any nontrivial $P, Q$, but there is no need to do so here.

It follows from (64) that $\# Z(1)_{n}=F_{n+1}$, the $(n+1)$ st Fibonacci number (defined by $F_{1}=F_{2}=1, F_{n+1}=F_{n}+F_{n-1}$ if $n \geq 2$ ). This explains 
our terminology "Fibonacci differential poset." More generally, the rank sizes $z_{n}(r)=\# Z(r)_{n}$ satisfy the recurrence

$$
\begin{gathered}
z_{n+1}(r)=r z_{n}(r)+z_{n-1}(r), \quad n \geq 1, \\
z_{0}(r)=1, \quad z_{1}(r)=r .
\end{gathered}
$$

We can ask what additional properties $Z(r)$ has besides those given by Theorem 5.3. Some of these properties are summed up in the following result.

5.4. Proposition. Let $r \in \mathbb{P}$. The following two conditions on a poset $P$ are equivalent.

(a) $P$ is an $r$-differential lattice (necessarily modular) such that every complemented interval has length $\leq 2$,

(b) $P$ is isomorphic to $Z(r)$.

Proof. We first show that $Z(r)$ is a lattice, which will then be modular by Proposition 1.3 and Theorem 5.3. By, e.g., [St6, Proposition 3.3.1], it suffices to show that (i) any two elements of $Z(r)$ have an upper bound, and (ii) any two elements of $Z(r)$ have a greatest lower bound (or meet). Let $x, y \in Z(r)$. Let $l$ be the larger of the lengths (= number of letters) of $x$ and $y$. Then it is easily seen that $2^{l}$ (i.e., the word $22 \cdots 2$, with $l 2$ 's in all) is an upper bound of $x$ and $y$. This proves (ii).

We will show that $x \wedge y$ exists by induction on $\rho(x)+\rho(y)$. This is clear for $\rho(x)+\rho(y)=0$, since $\widehat{O} \wedge \widehat{O}=\widehat{O}$. Assume true for $\rho(x)+\rho(y)<k$, and let $\rho(x)+\rho(y)=k$. If $x$ and $y$ are comparable, then $x \wedge y$ is the lesser of $x$ and $y$, so assume $x$ and $y$ are incomparable. If $x=1_{i} v$ then $x$ covers the unique element $v$. Hence $x \wedge y=v \wedge y$. Thus we may assume $x=2 u$, $y=2 v$. We claim that $(2 u) \wedge(2 v)=2(u \wedge v)$. It is easily seen that

$$
u^{\prime} \leq v^{\prime} \Leftrightarrow 2 u^{\prime} \leq 2 v^{\prime} \text {. }
$$

Hence $2(u \wedge v) \leq 2 u$ and $2(u \wedge v) \leq 2 v$, i.e., $2(u \wedge v)$ is a lower bound of $2 u$ and $2 v$. If $z=2 z^{\prime}$, then by (66) we have $z^{\prime} \leq u \wedge v$, so $z \leq 2(u \wedge v)$. If $z=1_{i} z^{\prime}$ and $z \leq 2 s$ for some $s \in Z(r)$, then it is easily seen that $z^{\prime} \leq s$, so $2 z^{\prime} \leq 2 s$. Thus since $1_{i} z^{\prime} \leq 2 u$ and $1_{i} z^{\prime} \leq 2 v$, we get $1_{i} z^{\prime} \leq 2 z^{\prime} \leq 2 u$ and $1_{i} z^{\prime} \leq 2 z^{\prime} \leq 2 v$. Hence $2 z^{\prime}$ is a lower bound for $2 u$ and $2 v$, so by what we have just shown $1_{i} z^{\prime} \leq 2 z^{\prime} \leq 2(u \wedge v)$. Therefore $(2 u) \wedge(2 v)=2(u \wedge v)$ as claimed, so $Z(r)$ is a modular lattice.

Now in a locally finite modular lattice, an interval $[x, y]$ is complemented if and only if $y$ is the join of elements covering $x$ (see [Bi, Theorem 14, p. 16 and Theorem 6,p. 88]). Thus to show that every complemented interval of $Z(r)$ has length $\leq 2$, it suffices to show that for any $x \in Z(r)$, there is a $y$ which covers every element of $Z(r)$ which covers $x$. But one sees easily from the definition of $Z(r)$ that $y=2 x$. Hence $(b) \Rightarrow(a)$.

We will show that $(\mathrm{a}) \Rightarrow(\mathrm{b})$ by proving by induction on $n$ that if $P$ satisfies (a) then the rank-selected subposet $P_{[0, n]}:=\{x \in P: \rho(x) \leq n\}$ is uniquely 
determined (up to isomorphism). Clearly $P_{[0,0]}$ is unique-it consists of the single element $\widehat{O}$. Assume that $P_{[0, n-1]}$ is uniquely determined. Let $x \in P_{n-2}$, and let $C^{+}(x)$ be the set of elements covering $x$. Since every complemented interval has length $\leq 2$, there must be an element $x^{\prime} \in P_{n}$ which covers every element of $C^{+}(x)$. These elements $x^{\prime}$ must be distinct, for if $x^{\prime}=y^{\prime}$ but $x \neq y$, then $x^{\prime}$ would cover two elements which do not cover a third element, contradicting modularity. After these elements $x^{\prime}$ are adjoined to $P$, every element $y \in P_{n-1}$ will cover as many elements as it has elements covering it. Thus by (D3) we must adjoin $r$ elements $y_{1}, y_{2}, \ldots, y_{r}$ covering $y$. If $y, z \in P_{n}$ and $y \neq z$, then again by modularity $y_{i} \neq z_{j}$. Thus $P_{n}$ is uniquely determined, so by induction $(\mathrm{a}) \Rightarrow(\mathrm{b})$.

Note that from the above proof we get another proof that $F(Z(r), q)=$ $\left(1-r q-q^{2}\right)^{-1}$. For we obtained one element $x^{\prime} \in P_{n}$ for each element $x \in$ $P_{n-2}$, and $r$ elements $y_{1}, \ldots, y_{r} \in P_{n}$ for each element $y \in P_{n-1}$. Hence

$$
\# P_{n}=r\left(\# P_{n-1}\right)+\left(\# P_{n-2}\right) \text {, }
$$

which together with the initial conditions $\# P_{0}=1$ and $\# P_{1}=r$ immediately yields (64).

There is a characterization of the $r$-differential posets $Y^{r}$ similar to Proposition 5.4.

5.5. Proposition. Let $r \in \mathbb{P}$. The following two conditions on a poset $P$ are equivalent.

(a) $P$ is an $r$-differential distributive lattice,

(b) $P$ is isomorphic to $Y^{r}$.

Proof. Corollary 1.4 asserts that $(\mathrm{b}) \Rightarrow(\mathrm{a})$. It is stated without proof in [St $2, \mathrm{p}$. 267] for $r=1$ and (in a more general context) in [St3, p. 225] that (a) $\Rightarrow(b)$. A proof appears in [St6, solution to Exercise 3.22(a), p. 180].

If $P$ is a locally finite poset with $\widehat{O}$, then for $x \in P$ let $e(x)=e(P, x)$ denote the number of maximal chains of the interval $[\widehat{O}, x]$. Young's lattice $Y$ (and hence $Y^{r}$ by a simple argument) has the property that for any partition $\lambda \in Y$, the number $e(\lambda)$ has a simple explicit formula. Namely, $e(\lambda)$ is the number of standard Young tableaux of shape $\lambda$ (usually denoted $f^{\lambda}$ or $f_{\lambda}$ ) and so is given by the Frame-Robinson-Thrall hook length formula [M, Example I.5.2, pp. 43 and 53; St2, Proposition 17.1]. A similar result holds for $Z(r)$. To prove this result for $Z(r)$, we will consider a certain poset $\mathrm{Fib}(r)$ related to $Z(r)$. First define a partial ordering on the alphabet $A_{r}=\left\{1_{1}, 1_{2}, \ldots, 1_{r}, 2\right\}$ by the condition that $1_{i}<2$ for all $i$ (and no other inequalities $a<b$ ).

5.6. Definition. Let $r \in \mathbb{P}$. Define a poset $\operatorname{Fib}(r)$ as follows. As a set, $\operatorname{Fib}(r)$ coincides with $A_{r}^{*}$. If $u=a_{1} a_{2} \cdots a_{j}$ and $v=b_{1} b_{2} \cdots b_{k}$ are two words in $A_{r}^{*}$, 
then define $u \leq v$ if $j \leq k$ and $a_{i} \leq b_{i}$ for $1 \leq i \leq j$. The poset $\operatorname{Fib}(r)$ is called the $r$-Fibonacci lattice.

With $r=1, \mathrm{Fib}(1)$ is the "Fibonacci lattice" discussed in [St3, §4]. Fib(1) is a distributive lattice, while it is not difficult to see that for $r \geq 2 \mathrm{Fib}(r)$ is join-distributive but not distributive. This means that every interval $[x, y]$ for which $y$ is a join of the elements of $[x, y]$ covering $x$ is a boolean algebra. See [E] or [St6, Exercises 3.19-3.20] for further information on join-distributive lattices (in the dual form of "meet-distributive lattices").

As discussed in [St3], if $x \in \operatorname{Fib}(1)$ then the interval $[\widehat{O}, x]$ is isomorphic to the lattice $J\left(T_{x}\right)$ of order ideals of a certain "dual tree" (as defined in [St6, p. 294]) $T_{x}$. It follows from [St $\left.1, \S 22\right]$ that

$$
e(\operatorname{Fib}(1), x)=\left(\# T_{x}\right) ! / \prod_{y \in T_{x}}\left(\# V_{y}\right),
$$

where $V_{y}=\left\{z \in T_{x}: z \geq y\right\}$. It is shown in [St3, Proposition 6], that the multiset of numbers $e(x)$, for $x \in \operatorname{Fib}(1)_{n}$, coincides with the multiset of products $\prod_{i \in S} i$, where $S$ ranges over all subsets of $\{1,2, \ldots, n-1\}$ containing no two consecutive integers.

Now consider $x \in \operatorname{Fib}(r)$. A maximal chain $\widehat{O}=x_{0}<x_{1}<\cdots<x_{n}=x$ in the interval $[\widehat{O}, x]$ becomes a maximal chain $\widehat{O}=\bar{x}_{0}<\bar{x}_{1}<\cdots<\bar{x}_{n}=\bar{x}$ of the interval $[\hat{O}, \bar{x}]$ of $\operatorname{Fib}(1)$, where $\bar{y}$ denotes the word $y$ with each $1_{i}$ changed to $1_{1}$. Let $t(w)$ denote the number of 2 's in the word $w$. Then there are exactly $r^{t(x)}$ maximal chains $\hat{O}=x_{0}<x_{1}<\cdots<x_{n}=x$ of the interval $[\widehat{O}, x]$ of $\operatorname{Fib}(r)$ such that $\widehat{O}=\bar{x}_{0}<\bar{x}_{1}<\cdots<\bar{x}_{n}=\bar{x}$ is a specified chain in Fib(1), since each 2 in $x$ will at some point when moving down the chain be converted to $1_{i}, 1 \leq i \leq r$. It follows that

$$
e(\operatorname{Fib}(r), x)=r^{t(x)} e(\operatorname{Fib}(1), \bar{x})=r^{t(x)}\left(\# T_{x}\right) ! / \prod_{y \in T_{x}}\left(\# V_{y}\right) .
$$

We now come to the connection between the posets $Z(r)$ and $\mathrm{Fib}(r)$ which yields a simple formula for $e(Z(r), x)$.

5.7. Proposition. The "identity" map $\phi: \mathrm{Fib}(r) \rightarrow Z(r)($ defined by $\phi(x)=x)$ is a rank-preserving bijection which preserves the function $e$, i.e.,

$$
e(\operatorname{Fib}(r), x)=e(Z(r), x), \quad \text { for all } x \in A_{r}^{*} .
$$

Proof. Clearly $\phi$ is a bijection, and it is easy to see that it is rank-preserving. Now the function $e(Z(r), x)$ is uniquely determined by the conditions

$$
e(Z(r), \hat{O})=1, \quad e(Z(r), x)=\sum_{y \in C^{-}(x)} e(Z(r), y), \quad \text { if } x>\widehat{O}
$$


where $C^{-}(x)$ is computed in $Z(r)$. Hence to show that $e(\operatorname{Fib}(r), x)=$ $e(Z(r), x)$ for all $x \in A_{r}^{*}$, it suffices to show that

$$
\begin{aligned}
& e(\operatorname{Fib}(r), \widehat{O})=1, \\
& e(\operatorname{Fib}(r), x)=\sum_{y \in C^{-}(x)} e(\operatorname{Fib}(r), y), \quad \text { if } x>0,
\end{aligned}
$$

where $C^{-}(x)$ is computed in $Z(r)$ (not in $\mathrm{Fib}(r)$, where (68) is obvious).

Of course $e(\operatorname{Fib}(r), \widehat{O})=1$ is trivial. We show the second equality of (68) first for the case $r=1$. We will write $A_{1}=\{1,2\}$ instead of $\left\{1_{1}, 2\right\}$, and $e(y)$ instead of $e(\operatorname{Fib}(1), y)$.

Suppose $x=2^{j} 1 v \in Z(1)$. We need to show

$$
e(x)=\sum_{i=1}^{j} e\left(2^{i-1} 12^{j-i} v\right)+e\left(2^{j} v\right) .
$$

We remarked above that $[\widehat{O}, x] \cong J\left(T_{x}\right)$ for a certain dual tree $T_{x}$. Specifically, $T_{x}$ is defined by the conditions that (a) if $j=0$, then the root $\widehat{O}$ has the unique dual subtree $T_{v}$, and (b) if $j>0$, then $\widehat{O}$ has the two dual subtrees $T_{1}$ and $T_{2^{j-1} 1 v}$. Now since $[\widehat{O}, x] \cong J\left(T_{x}\right)$, the number of maximal chains $e(x)$ in the interval $[\widehat{O}, x]$ is equal to the number of linear extensions $e\left(T_{x}\right)$ of the poset $T_{x}$ [St6, p. 110]. Suppose $P$ is a finite poset, and $Q$ is a subset of $P$ satisfying the condition that for any $y \in P-Q$, either (a) $y<z$ for all $z \in Q$, (b) $y>z$ for all $z \in Q$, or (c) $y$ is incomparable to $z$ for all $z \in Q$. Then $e(P)$ is unchanged by "dualizing $Q$ " inside $P$, i.e., $e(P)=e\left(P^{\prime}\right)$, where $P^{\prime}$ is the poset with the same elements and same relations as $P$, except that if $y, z \in Q$ and $y<z$ in $P$, then $y>z$ in $P^{\prime}$. Consider the case of $P=T_{x}$, where $x=2^{j} 1 v \in Z(1)$. Choose $Q=T_{y}$, where $y=1 v$. The poset $P^{\prime}$ has $j+1$ maximal elements $z_{1}, \ldots, z_{j+1}$, and

$$
e\left(P^{\prime}\right)=\sum_{i} e\left(P^{\prime}-z_{i}\right)
$$

Now in each $P^{\prime}-z_{i}$ choose a new $Q_{1}=\left(P^{\prime}-z_{i}\right) \cap Q$. Then dualizing $Q_{1}$ inside $P^{\prime}-z_{i}$ yields the poset $\left(P^{\prime}-z_{i}\right)^{\prime}=P-z_{i}$, and $e\left(P^{\prime}-z_{i}\right)=e\left(P-z_{i}\right)$. But the posets $P-z_{i}$ are given by $T_{y}$ where $y=2^{i-1} 12^{j-i} v$ for $1 \leq i \leq j$, and $y=2^{j} v$. Hence (69) follows from (70).

There remains the case $x=2^{j}$. But this is even easier than the case $x=$ $2^{j} 1 v$, since we do not need to dualize a subset of $T_{x}$. (In other words, the subsets $T_{y}$ of $T_{x}$, where $y=2^{i-1} 12^{j-i}$, are just $T_{x}$ with one of its maximal elements removed.) Hence

$$
e\left(2^{j}\right)=\sum_{i=1}^{j} e\left(2^{i-1} 12^{j-i}\right),
$$

and the proof for $r=1$ is complete. 
Now let $r$ be arbitrary. We will show that

$$
e(Z(r), x)=r^{t(x)} e(Z(1), \bar{x}),
$$

in the notation of $(67)$. Since $e(Z(1), \bar{x})=e(\operatorname{Fib}(1), \bar{x})$, it then follows from (67) that $e(Z(r), x)=e(\operatorname{Fib}(r), x)$. The proof of (71) is similar to that of (67). If $\hat{O}=x_{0}<x_{1}<\cdots<x_{n}=x$ is a maximal chain of the interval $[\widehat{O}, x]$ of $Z(r)$, then $\widehat{O}=\bar{x}_{0}<\bar{x}_{1}<\cdots<\bar{x}_{n}=\bar{x}$ is a maximal chain of the interval $[\widehat{O}, \bar{x}]$ of $Z(1)$. Conversely, there are exactly $r^{t(x)}$ maximal chains of the interval $[\widehat{O}, x]$ of $Z(r)$ which correspond to a specified maximal chain of the interval $[\hat{O}, \bar{x}]$ of $Z(1)$, since each 2 appearing in $x$ will at some point when moving down the chain be converted to $1_{i}, 1 \leq i \leq r$. There are $r$ choices for each 2 ; hence $2^{t(x)}$ choices in all, as claimed.

We could have shown that

$$
e(Z(r), x)=r^{t(x)}\left(\# T_{x}\right) ! / \prod_{y \in T_{x}}\left(\# V_{y}\right)
$$

directly by induction on $\rho(x)$, but we wanted to point out the connection between $Z(r)$ and $\mathrm{Fib}(r)$. One reason for doing so is that in [St3, p. 228] it is asked (implicitly) if it is just a coincidence that $\alpha(0 \rightarrow n)$, as well as $\alpha(0 \rightarrow n \rightarrow 0)$, is the same for both $Y$ and Fib(1). We now have an explanation for this "coincidence," namely, the connection between $\mathrm{Fib}(1)$ and $Z(1)$ given by Proposition 5.7, together with the fact that $Z(1)$ is a 1-differential poset.

It is natural to ask whether $\operatorname{Fib}(r)$ and $Z(r)$ share additional properties besides the values of $\alpha(0 \rightarrow n)$ and $\alpha(0 \rightarrow n \rightarrow 0)$. Not surprisingly, $\operatorname{Fib}(r)$ does not possess most of the enumerative properties of $r$-differential posets. For instance, the number $\delta(n)$ of Hasse walks from $\widehat{O}$ of length $n$ do not agree for $\operatorname{Fib}(r)$ and $Z(r)$.

\section{OPEN PROBLEMS}

An obvious question at this point is the following.

Problem 1. Classify all $r$-differential posets.

D. Wagner has described a very general method for constructing differential posets which make it unlikely that Problem 1 has a reasonable answer. The following special case of Wagner's construction suffices to show that for each $r$ there are infinitely many irreducible $r$-differential posets. Let $P$ be a graded poset of rank $n$. Define the reflection extension $P^{+}$of $P$ to be the poset of rank $n+1$ which coincides with $P$ for ranks $\leq n$ (i.e., $P_{[0, n]}^{+}=P$, in the notation of $\S 4)$, and has an element $x^{*} \in P_{n+1}^{+}$for each $x \in P_{n-1}$, with the cover relations $x^{*}$ covers $y \in P_{n}$ if $y$ covers $x \in P_{n-1}$. (This construction is closely related to the "fundamental construction" of [G-H-J].) Define $E_{r}(P)$ to 
be the poset obtained from $P^{+}$by adjoining $r$ additional elements above each element $x \in P_{n}$. If $P$ satisfies (D1), (D2) for elements $x$ and $y$ of rank $<n$, and (D3) for elements $x$ of rank $<n$, then we call $P$ a partial $r$-differential poset of rank $n$. It is then easily seen that $E_{r}(P)$ is a partial $r$-differential poset of rank $n+1$. There follows:

6.1. Proposition. Let $P$ be a partial $r$-differential poset of rank $n$. Let

$$
Q=E_{r}^{\infty}(P)=\lim _{j \rightarrow \infty} E_{r}^{j}(P),
$$

where $E_{r}^{j}(P):=E_{r}\left(E_{r}^{j-1}(P)\right)$. Then $Q$ is an $r$-differential poset with $Q_{[0, n]}=$ $P$.

If we pick $P=Y_{[0, n]}^{r}$ in Proposition 6.1 (where $Y$ is Young's lattice), then $E_{r}^{\infty}(P)$ can be seen to be an irreducible $r$-differential poset; and we obtain nonisomorphic posets for different values of $n$ (with a few exceptions for small $n)$. If we start with $P$ equal to a single point, then $E_{r}^{\infty}(P) \cong Z(r)$.

In view of the seemingly wide variety of differential posets, we should perhaps replace Problem 1 by the following less precise question.

Problem 2. Find "interesting" examples of $r$-differential posets. For instance, are there examples with characterizations similar to those in Propositions 5.4 and 5.5? Are there any irreducible differential lattices besides $Y$ and $Z(r)$ ?

Another class of problems (numbers 3-5) is concerned with extending properties of Young's lattice to arbitrary differential posets $P$. For instance, let $0=$ $a_{0}<a_{1}<a_{2}<\cdots<a_{k}$ be positive integers, and let $\alpha\left(P ; n, n+a_{1}, \ldots, n+a_{k}\right)$ be the number of chains $x_{0}<x_{1}<\cdots<x_{k}$ in $P$ for which $\rho\left(x_{i}\right)=n+a_{i}$, $0 \leq i \leq k$. It is known [St6, Exercise 4.21(a); Bu, Lemma 6.3] that there is a rational function $R(q)$ (depending on $a_{0}, a_{1}, \ldots, a_{k}$ ) such that

$$
\sum_{n \geq 0} \alpha\left(Y ; n, n+a_{1}, \ldots, n+a_{k}\right) q^{n}=R(q) F(Y, q) .
$$

Problem 3. Let $P$ be an $r$-differential poset. Is there a rational function $R(q)$ (depending on $a_{0}, a_{1}, \ldots, a_{k}$ and $P$ ) such that

$$
\sum_{n \geq 0} \alpha\left(P ; n, n+a_{1}, \ldots, n+a_{k}\right) q^{n}=R(q) F(P, q) ?
$$

MacMahon's famous generating function [St2, Corollary 18.2] (with an obvious misprint) for $k$-rowed plane partitions may be written as follows:

$$
\sum q^{\rho\left(x_{1}\right)+\cdots+\rho\left(x_{k}\right)}=\prod_{i \geq 1}\left(1-q^{i}\right)^{-\min (i, k)},
$$

where $k \in \mathbb{N}$ is fixed, and the sum runs over all $k$-element multichains $\hat{O} \leq$ $x_{0} \leq x_{1} \leq \cdots \leq x_{k}$ in $Y$.

Problem 4. Is there a "nice" formula for the left-hand side of (72) when $P$ is an arbitrary $r$-differential poset? In particular, does it equal $R(q) F(P, q)^{k}$ for some rational function $R(q)$ (depending on $P$ and $k$ )? 
Problem 5. Let $P$ be an $r$-differential poset, and let $x \in P_{n}$. Is it always true that for some integer $0 \leq m(x) \leq n$ which is determined by $x$ is some "simple" way, we have that $e(x)=r^{m(x)} e_{0}(x)$ where $e_{0}(x) \mid(n !)$ ? More specifically, can we define in a simple way positive integers $h_{1}(x), h_{2}(x), \ldots, h_{n}(x)$ such that

$$
e(x)=r^{m(x)} n ! / \prod_{1}^{n} h_{i}(x) ?
$$

(For the posets $Y^{r}$ we can always take $m=0$; for $Z(r), m$ is equal to the number $t(x)$ of 2 's in $x$ by (67) and Proposition 5.7.)

Finally we mention five miscellaneous problems.

Problem 6. Fix $r \in \mathbb{P}$. What is the greatest (respectively, least) number $\alpha(n)$ of elements of rank $n$ that an $r$-differential poset can have? It seems plausible that the extreme values are achieved by $Z(r)$ and $Y^{r}$, respectively. Along the same lines, given that $p_{j}=\# P_{j}$ for some $j$, what is the smallest (respectively, largest) cardinality of $P_{j+1}$ ? Do we always have $p_{j+1} \leq r p_{j}+p_{j-1}$ ? Do we always have $p_{j+1}>p_{j}$, except when $r=1$ and $j=0$ ?

Problem 7. What is the automorphism group Aut $Z(r)$ of the lattice $Z(r)$ ?

Regarding Problem 7, clearly the symmetric group $S_{r}$ acts on $Z(r)$ by permuting the letters $1_{1}, 1_{2}, \ldots, 1_{r}$. However, $Z(1)$ has (at least) an additional automorphism $\omega$, defined by

$$
\omega(v 11)=v 2, \quad \omega(v 2)=v 11, \quad \omega(w)=w, \quad \text { otherwise. }
$$

We suspect that Problem 7 should not be too difficult.

Problem 8. Fix $r \in \mathbb{P}$. Is there a "natural" sequence $\mathscr{A}_{0} \subset \mathscr{A}_{1} \subset \cdots$ of semisimple algebras $\mathscr{A}_{n}$ (over $\mathbb{C}$, say) whose relationship to $Z(r)$ is analogous to the relationship between the group algebras $\mathbb{C} S_{0} \subset \mathbb{C} S_{1} \subset \cdots$ of the symmetric groups $S_{n}$ and Young's lattice? More precisely, we would have $\operatorname{dim} \mathscr{A}_{n}=r^{n} n$ !, and the irreducible representations $\rho_{x}$ of $\mathscr{A}_{n}$ would be indexed by elements $x \in Z(r)_{n}$. Moreover, $\operatorname{dim} \rho_{x}=e(x)$, and the restriction of $\rho(x)$ to $\mathscr{A}_{n-1}$ decomposes as

$$
\left.\rho_{x}\right|_{\mathscr{Q}_{n-1}}=\sum_{y \in C^{-}(x)} \rho_{y} .
$$

(Condition (73) is equivalent to saying that the bipartite graph $Z(r)_{n-1, n}$ is the Brattelli diagram of the pair $\mathscr{A}_{n-1} \subset \mathscr{A}_{n}$.) The existence of abstract algebras $\mathscr{A}_{n}$ with the desired properties is easy to see; in fact, it follows from the "fundamental construction" of [G-H-J] that $\mathscr{A}_{n+1} \cong\left(\right.$ End $\left._{\mathscr{A}_{n-1}} \mathscr{A}_{n}\right) \oplus \mathscr{A}_{n}^{r}$, where End $\mathscr{A}_{n-1} \mathscr{A}_{n}$ is defined by a certain embedding $\mathscr{A}_{n-1} \subset \mathscr{A}_{n}$ corresponding to the Brattelli diagram $Z(r)_{n-1, n}$. What is wanted is a "natural" combinatorial definition of $\mathscr{A}_{n}$.

Problem 9. Characterize in some reasonable way those bipartite graphs which are isomorphic to a rank-selected subposet $P_{j-1, j}$ of some $r$-differential poset 
$P$. (The axioms (D1)-(D3), as well as Theorem 4.14, lead to some necessary conditions.)

Problem 10. Suppose that $P$ and $Q$ are $r$-differential posets and that for some $j \in \mathbb{P}$ we have $P_{j-1, j} \cong Q_{j-1, j}$. Is it true that $P_{[0, j]} \cong Q_{[0, j]}$ ? (See Corollary 4.5.)

\section{REFERENCES}

[B-C-L] M. Behzad, G. Chartrand, and L. Lesniak-Foster, Graphs \& Digraphs, Wadsworth, Belmont, CA, 1979.

[Be] A. Berele, A Schensted-type correspondence for the symplectic group, J. Combin. Theory A 43 (1986), 320-328.

[Bi] G. Birkhoff, Lattice theory, 3rd ed., Amer. Math. Soc., Providence, RI, 1967.

[B-M] A. Bondy and U. S. R. Murty, Graph theory with applications, North-Holland, New York, 1976.

[Bu] L. Butler, Rational generating functions for enumerating chains of partitions, preprint.

[C] L. Comtet, Advanced combinatorics, Reidel, Dordrecht/Boston, 1974.

[C-D-S] D. M. Cvetkovic, M. Doob, and H. Sachs, Spectra of graphs, Academic Press, New York, 1980.

[E] P. Edelman, Abstract convexity and meet-distributive lattices, Proc. AMS-IMS-SIAM Conf. on Combinatorics and Ordered Sets, Arcata, 1985 (I. Rival, ed.), Contemp. Math. 57 (1986), 127-150.

[G] R. J. Glauber, Some notes on multiple-boson processes, Phys. Rev. 84 (1951), 395-400.

[G-H-J] F. M. Goodman, P. de la Harpe, and V. Jones, Coxeter diagrams and towers of algebras, Chapter 2, Towers of multi-matrix algebras (preliminary version), MSRI book series, Springer, New York (to appear).

[H-W] P. Hanlon and D. Wales, On the decomposition of Brauer's centralizer algebras, J. Algebra (to appear).

[K] G. Kreweras, Sur une classe de problèmes de dénombrement liés au trellis des partitions des entiers, Cahiers du BURO, vol. 6, 1965.

[Ku] J. P. S. Kung, Radon transforms in combinatorics and lattice theory, Contemp. Math. 57 (1986), 33-74.

[M] I. G. Macdonald, Symmetric functions and Hall polynomials, Oxford Univ. Press, Oxford, 1979.

[Mi] W. Miller, Jr., Lie theory and special functions, Academic Press, New York and London, 1968.

[M-M] M. Marcus and H. Minc, A survey of matrix theory and matrix inequalities, Prindle, Weber, \& Schmidt, Boston, 1964.

[P] R. A. Proctor, Representations of $\mathrm{sl}(2, \mathbb{C})$ on posets and the Sperner property, SIAM J. Algebraic Discrete Methods 3 (1982), 275-280.

[S-S] B. Sagan and R. Stanley, Robinson-Schensted algorithms for skew tableaux (to appear).

[St1] R. Stanley, Ordered structures and partitions, Mem. Amer. Math. Soc., no. 119, Amer. Math. Soc., Providence, RI, 1971.

[St2] _ Theory and application of plane partitions, Parts 1 and 2, Studies in Applied Math. 50 (1971), 167-188, 259-279.

[St3] _ The Fibonacci lattice, Fibonacci Quart. 13 (1975), 2.15-232.

$[\mathrm{St} 4] \ldots$, The stable behavior of some characters of $\operatorname{SL}(n, \mathbb{C})$, Linear and Multilinear Algebra 16 (1984), 3-27.

[St5] _ , Quotients of Peck posets, Order 1 (1984), 29-34. 
[St6] _ _ Enumerative combinatorics, vol. I, Wadsworth and Brooks/Cole, Pacific Grove, CA, 1986.

[St7] _ Weyl groups, the hard Lefschetz theorem, and the Sperner property, SIAM J. Algebraic Discrete Methods 1 (1980), 168-184.

[St8] _ _ Variations on differential posets, Invariant Theory and Tableaux (D. Stanton, ed.), The IMA Volumes in Mathematics and Its Applications, Springer, New York (to appear).

[Su] S. Sundaram, On the combinatorics of representations of $\operatorname{Sp}(2 n, \mathbb{C})$, Ph.D. thesis, M.I.T., 1986.

Department of Mathematics, Massachusetts Institute of Technology, Cambridge, MA 02139 